\title{
The Zur regulon of Corynebacterium glutamicum ATCC 13032
}

Jasmin Schröder ${ }^{1}$, Nina Jochmann ${ }^{1,2}$, Dmitry A Rodionov ${ }^{3,4}$, Andreas Tauch $^{{ }^{*}}$

\begin{abstract}
Background: Zinc is considered as an essential element for all living organisms, but it can be toxic at large concentrations. Bacteria therefore tightly regulate zinc metabolism. The Cg2502 protein of Corynebacterium glutamicum was a candidate to control zinc metabolism in this species, since it was classified as metalloregulator of the zinc uptake regulator (Zur) subgroup of the ferric uptake regulator (Fur) family of DNA-binding transcription regulators.

Results: The cg2502 (zur) gene was deleted in the chromosome of C. glutamicum ATCC 13032 by an allelic exchange procedure to generate the zur-deficient mutant C. glutamicum JS2502. Whole-genome DNA microarray hybridizations and real-time RT-PCR assays comparing the gene expression in C. glutamicum JS2502 with that of the wild-type strain detected 18 genes with enhanced expression in the zur mutant. The expression data were combined with results from cross-genome comparisons of shared regulatory sites, revealing the presence of candidate Zur-binding sites in the mapped promoter regions of five transcription units encoding components of potential zinc ABC-type transporters (cg0041-cg0042/cg0043; cg2911-cg2912-cg2913), a putative secreted protein (cg0040), a putative oxidoreductase (cg0795), and a putative P-loop GTPase of the COG0523 protein family (cg0794). Enhanced transcript levels of the respective genes in C. glutamicum JS2502 were verified by real-time RT-PCR, and complementation of the mutant with a wild-type zur gene reversed the effect of differential gene expression. The zinc-dependent expression of the putative cg0042 and cg2911 operons was detected in vivo with a gfp reporter system. Moreover, the zinc-dependent binding of purified Zur protein to double-stranded 40-mer oligonucleotides containing candidate Zur-binding sites was demonstrated in vitro by DNA band shift assays.

Conclusion: Whole-genome expression profiling and DNA band shift assays demonstrated that Zur directly represses in a zinc-dependent manner the expression of nine genes organized in five transcription units.

Accordingly, the Zur (Cg2502) protein is the key transcription regulator for genes involved in zinc homeostasis in $C$. glutamicum.
\end{abstract}

\section{Background}

Corynebacterium glutamicum is a gram-positive soil bacterium that is well-established for the industrial production of several $\mathrm{L}$-amino acids [1,2]. The complete genome sequence of the type strain C. glutamicum ATCC 13032 is available [3], and it was screened by bioinformatic tools to predict the repertoire of DNAbinding transcription regulators in this organism $[4,5]$. Transcription regulators represent key components in the control of bacterial gene expression and permit the cell to sense and respond to environmental changes [6].

\footnotetext{
* Correspondence: tauch@cebitec.uni-bielefeld.de

${ }^{1}$ Institut für Genomforschung und Systembiologie, Centrum für Biotechnologie, Universität Bielefeld, D-33615 Bielefeld, Germany
}

Amongst others, metal ion homeostasis in bacterial cells is tightly regulated by specific metal-sensing transcription regulators. These metalloregulatory proteins, in principle, sense the intracellular levels of specific metal ions by binding them to a metal binding site, which leads to conformational changes affecting the regulator's ability to bind operator sites in regulatory DNA regions [7]. Prominent protein families of metalloregulators are DtxR [8], MerR [9], SmtB/ArsR [10], and Fur [11]. The ferric uptake regulator Fur was originally described as iron-sensing repressor of genes involved in siderophore biosynthesis and iron transport in Escherichia coli $[12,13]$, but Fur also activates the expression of many genes by either direct or indirect mechanisms and can 
be regarded as global transcription regulator of iron homeostasis in E. coli [14]. Numerous studies indicated a tremendous diversity in metal selectivity and biological function within the Fur protein family that can be divided into sensors of iron (Fur), manganese (Mur), nickel (Nur), and zinc (Zur) [14].

Zinc is considered an essential nutrient for all living organisms. As zinc can be toxic at large concentrations [15], zinc uptake, efflux, storage, and metabolism is in general tightly regulated in bacteria [16]. During our work on reconstructing the transcriptional regulatory network of C. glutamicum [5,17], we recognized the Cg2502 protein as candidate to control the zinc metabolism in this species, since it was classified as DNA-binding transcription regulator of the Fur family [4] and iron metabolism is under global control of the dual regulator Cg2103, a member of the DtxR protein family [18]. In this study, comparative whole-genome DNA microarray hybridizations revealed a set of differentially expressed genes that are under transcriptional control by Cg2502 (now named Zur). Comparative genomic analysis of Zur regulons in actinobacteria detected candidate Zur-binding sites within the mapped promoter regions of potential target genes in C. glutamicum ATCC 13032. The DNA binding of Zur to these operator sites occurred in a zinc-dependent manner and was verified by DNA band shift assays, providing clear evidence that Zur is involved in zinc-dependent transcriptional regulation of gene expression in C. glutamicum ATCC 13032.

\section{Results}

\section{Annotation of the corynebacterial zinc uptake regulator} Zur

The Cg2502 (Zur) protein of C. glutamicum ATCC 13032 has a predicted size of 144 amino acids, a theoretical molecular mass of $15.7 \mathrm{kDa}$ and belongs to the small core set of 24 transcription regulators that were detected in all hitherto sequenced corynebacterial genomes $[5,19]$. Protein domain predictions performed with the SUPERFAMILY [20] and the Conserved Domain Database tools [21] showed that the Zur protein contains an amino-terminal helix-turn-helix motif of the winged-helix type and is a member of the Zur (zinc uptake regulator) subgroup of the Fur (ferric uptake regulator) family of metalloregulatory proteins [14]. According to BLASTP data [22], the C. glutamicum Zur protein revealed high amino acid sequence similarities to orthologous proteins encoded in other sequenced corynebacterial genomes, ranging from $56 \%$ to $80 \%$ identical amino acids (Fig. 1A). Furthermore, Zur orthologues in other actinobacteria are well conserved and corroborated by the phylogenetic tree for these proteins (Fig. 1B). The Zur orthologue in Mycobacterium tuberculosis H37Rv (57\% identity with Cg2502) is the zinc metalloregulator FurB, whose crystal structure has been elucidated recently [23]. The multiple alignment of Zur proteins from actinobacteria demonstrates the conservation of all amino acid residues forming three distinct zinc binding sites in the FurB protein (Fig. 1A). The zinc binding site 1 is surrounded by conserved aspartate, cysteine and histidine residues, whereas the zinc binding site 2 is represented by a cluster of four cysteines. The putative zinc binding site 3 is build by three histidines and one glutamate, but the exact biological function of this protein site remains to be determined [23]. These structural protein data strongly suggested that the Cg2502 (Zur) protein of C. glutamicum is a zinc-binding protein and involved in the transcriptional regulation of zinc metabolism in this species.

According to comparative genomic analysis, the cg2502 (zur) gene of C. glutamicum ATCC 13032 is located in a conserved gene region in all hitherto sequenced corynebacterial genomes (Fig. 2A). In the genomes of C. glutamicum, C. efficiens, C. diphtheriae, C. aurimucosum, and C. accolens, all representing members of the main lineage of the genus Corynebacterium $[24,25]$, the zur gene is located downstream of another regulatory gene $(z n r)$ encoding a putative metal-sensing transcription regulator of the $\mathrm{SmtB} / \mathrm{ArsR}$ protein family $[4,10]$. In genomes of corynebacteria belonging to the $C$. jeikeium and C. kroppenstedtii branches, the overall location of the zur gene is also conserved, but an orthologue of $z n r$ is lacking in front of the zur coding region (Fig. 2A). Since the orthologous protein of $\mathrm{Znr}$ from $M$. tuberculosis $\mathrm{H} 37 \mathrm{Rv}$ (Rv2358) is apparently involved in zinc-dependent transcriptional (auto)regulation of the rv2358-furB operon [26], the homologous znr-zur gene region of C. glutamicum ATCC 13032 may also encode the regulatory switches involved in controlling the zinc homeostasis in this organism.

Transcriptional organization of the znr-zur gene region in C. glutamicum

Operon predictions for C. glutamicum ATCC 13032 suggested that the znr-zur genes are expressed as bicistronic transcript [27]. To provide experimental support for this prediction, the transcription of the $z n r-z u r$ region was analyzed by marker gene expression using the green fluorescent protein encoded on the promoterprobe vector pEPR1 [28]. Both the $z n r$ upstream region and the znr-zur intergenic region were tested for promoter activity in E. coli DH5 $\alpha$ MCR and C. glutamicum ATCC 13032 (Fig. 3). For this purpose, a 141-bp DNA fragment covering the $z n r$ upstream region and a 107bp DNA fragment containing the 40-bp $z n r-z u r$ intergenic region were amplified by PCR and cloned in front of the promoterless $g f p$ gene present on pEPR 1 . The expression of $g f p$ was detected by fluorescence microscopy only with a pEPR1 derivative containing the $z n r$ 


\section{A)}

\section{cg2502}

CE2180

DIP1710

cauri 1801

HMPREF $0276 \quad 173$

ckrop $1315^{-}$

jk061̄

cur 1324

MT furB

MMĀR 3669

Mvan 3820

MSME $\bar{G} 4482$

SCO2508

Tfu 0856

PPA 0048

Lxx25010

BL1128

cg2502

CE2180

DIP1710

Cauri 1801

HMPREF 02761739

ckrop 1315

jk0612

cur_1324

MT furB

MMĀR 3669

Mvan-3820

MSMEḠ 4482

$\mathrm{SCO} 2508$

Tfu 0856

PPAO 948

Lx 25010

BL1128

DNA-binding domain

LGVRSTRQRKAVIDVLEE IDNFASAKE I HHELSTREHNVGLTTVYRTLQSLADIGAVDVLTVTGGETL 81 LGVRSTRQRKAVVDILEE IDNFASAKAI HAELNAREHSVGLTTVYRTLQSLAEIGAVDVLTVTGGETL 108 LGVRSTRQRTAVVGVLKDLDYFASAKVIHQELTKRDLKVGLTTVYRTLQSLSEIEAVDVLHMSNGETL 79 LGARNTRQRTAVVEVLRSLDKFVSAKDIHQALLDGDQKVGLTTVYRTLQSLSEIEAVDALHMPSGETL 77

LGSRNTKORTAVVEVLRD IDKFASAKE I YHOLOEROEKVGLTTVYRTLOSLSD IDAVDALHMPNGETL 80 IGIRSTRQRAAVEDTLADDLPRFASAGDIHRRIVDKGESVGLTTVYRTLOALADAGEVDVLNSSLGGEAL 77 IGQRNTRQRRAVAELLEGLTTFSSAQD I HQKLVAQDHKVGLTTVYRTLQQMAEAGT IDTLYDDSGETL 79 IGQRNTRQRAAVVAMLEQVEEFASAQDIHARLVAAGEKVGLTTVYRTLQQMSAMGAIDTLNNESGETL 115 AGVRSTRQRAAISTLLETLDDFRSAQELHDELRRRGENIGLTTVYRTLQSMASSGLVDTLHTDTGESV 71 TSVRSTRORAAISAILETVDDFRSAOELHDELRRRGENIGLTTVYRTLOSMASAGTVDTLRTDTGESV 71 GAVRSTRQRAAISALLENIEDFRSAQELHDELRRRGEGIGLTTVYRTLQQMAAAGVVDTLRTDTGESV 70 GAVRSTRQRAAIADLLNE TEGFRSAQELHDELRRRGEGIGLTTVYRTLQAMATAGVVDTLRTDTGESV 70 VKGRATRQRAAVSAALQEVEEFRSAQELHDMLKHKGDAVGLTTVYRTLQSLADAGEVDVLRTAEGESV 75 ---MSSROREAVROALHKSNGFRSAODLYASLRADGAKIGLTTVYRALOALTDAGEVDVLMTDEGEAV 64 NRFRTTKORLAIRALFDDEPFFLTAOOVHDOLRNRGDOVGLATVYRNLOTMAEDGELDAIRAEDGEMT 70 MVKRNTWQREAVREALSSTEGFISAQGLHLRLHEAGSPIGLATVYRALADLAAEGDADSLQSPEGESL 68 HIERQTKQKDAIRAALAD CEEFISAQDLHRRLEDEGSKIGLATVYRQLNALADAGAADTIR-LDGQQL 74

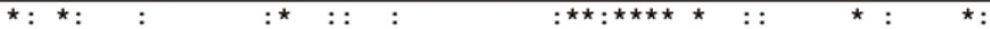

YROCHDEGHHHHLVCTNCGRTVE IDGG-PVETWAOE IATKNGFALSSHEAE IFGLCADCKEKVT---- 144 YROCHDE GHHHHLVCTOCGRTEE IDGG-PVERWAGEVARTHGF SVSSHEAE IFGLCPACRPDTVDGOA 175 YRHCLSDEHHHHLVCTECGRTVE IDGG-PVEKWAKEVAQLHGFQVTGHDAE IYGLCESCSAATE---- 142 YRHCESDAHHHHLVCTKCGRTEE IDGG-PIEEWASSVAKEHDFELTGHDAE IFG ICAECRA------- 137 YRHCETDAHHHHLVCTKCGRTEE IDGG-PIEKWASAVAAE YNFELTGHDAE IFGVCSOCAAAQ----- 142 YRKCNSS THHHHLVCTNCGAAVE IDGG-PVEEWSQQAADEHGYVLTGHTAEVFGLCPRCHGKKS---- 140 YRACATDDHHHHLVCT SCRRTVE IDGG-PVEDWAAETAKKFGYQKSGHTAE IFGLCGDCQKKSAS--- 143 YRRCAMD THHHHLVCNDCRTTVE IDGG-PVEDWARDVAKKYGFQKTGHTADVFGLCADCAAKKTQKN- 181 YRRCSE-HHHHHLVCRSCGSTIEVGDH-EVEAWAAEVATKHGF SDVSHT IE IFGTCSDCRS------- 130 YRRCSO-HHHHHLVCRSCGST IEVADH-EVETWAANVAARHGFSDVSHT IE IFGTCSDCRRPGAAALR 137 YRRCSE-HHHHHLVCRACGCTVEIQGG-QVETWAAEVAHEHGFSDVSHT IE IFGICSNCA---AGN-- 131 YRRCSE-HHHHHLVCRSCGSTVEVSGG-DVETWAAQVARD HGFSDVSHT IE IFGVCGDCVERPAGNQT 136 YRRCSTGDHHHHLVCRACGKAVEVEGP-AVEKWAEAIAAEHGYVNVAHTVE IFGTCADCAGASGG--- 139 YRACSTPTHHHHLVCRDCGKAVE IEGP-AVESWADELAAQHGFVDLTHTLELFGTCSDCAAAKPRT-- 129 YRRCSS-AHHHHLVCRNCGKAVE IGPEKILEDWTRDIAAKYEFSETGHELELFG ICSECS-----AVH 132 YRACTS-GHHHHLICRNCGLTVEIEAD-EVEAWARSAAADHGFSQVQHVVDVFGLCAGCS-AKADLAA 133 FRLCGDDGHHHHLVCRRCGKTVEIDP--PSEAWLRKVADGHGFTVESHTLEVFGLCSDCQKEQKAQKA 140

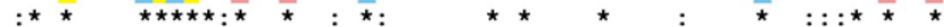

B) cg2502 Corynebacterium glutamicum ATCC 13032

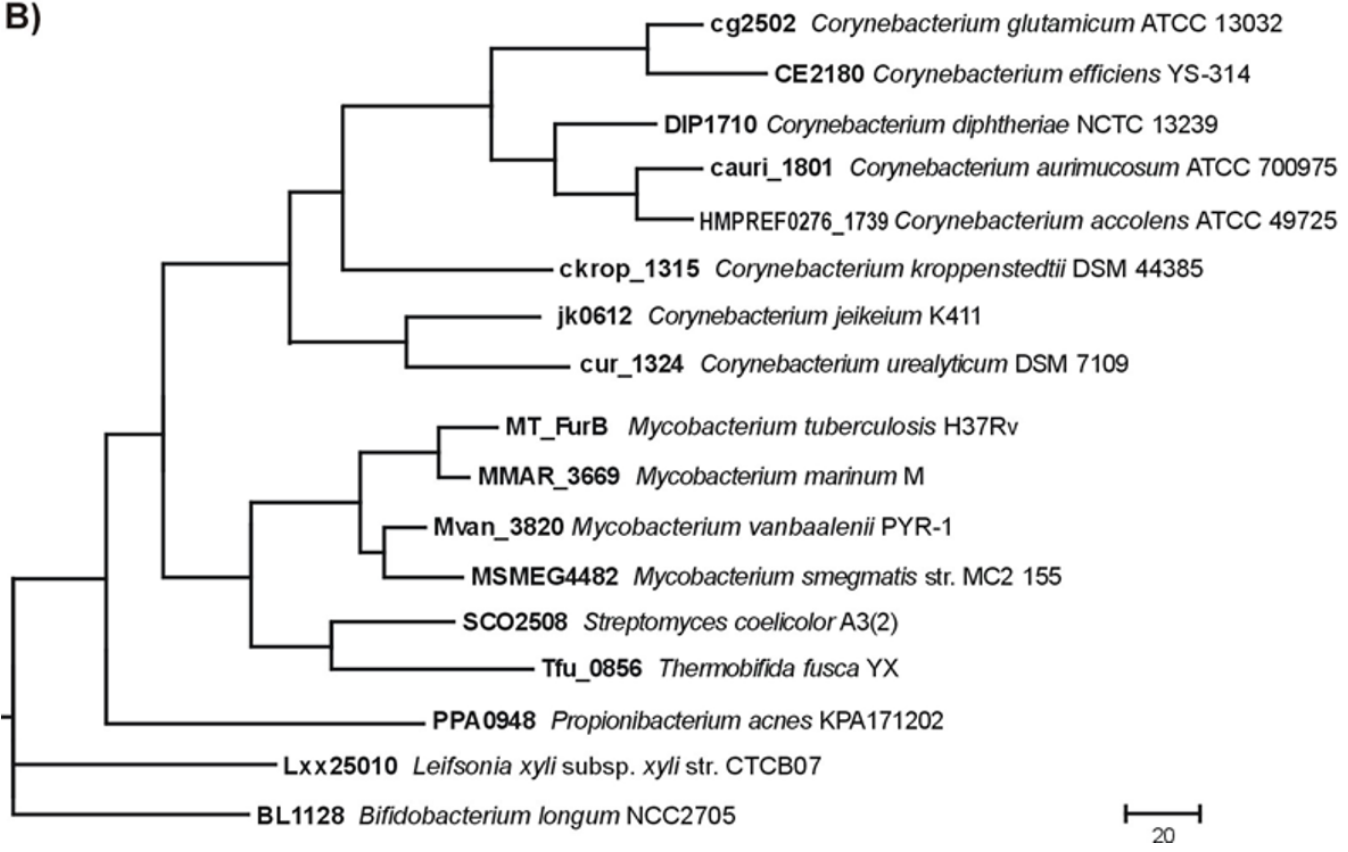

20

Figure 1 Comparative analysis of Zur proteins from actinobacteria. (A), Multiple amino acid sequence alignment of actinobacterial Zur proteins, including FurB from M. tuberculosis H37Rv. The winged-helix DNA binding domain is highlighted in grey. Three zinc binding sites (Zn 1 to $\mathrm{Zn} 3$ ) deduced from the crystal structure of the mycobacterial FurB protein [23] are specifically coloured. Zn 1 (yellow): Asp-71, Cys-85, His-91, and His-93; Zn 2 (red): Cys-96, Cys-99, Cys-136, and Cys-139; Zn 3 (blue): His-90, His-92, Glu-111, and His-128 (according to the C. glutamicum protein positions). (B), Maximum likelihood phylogenetic tree of Zur protein orthologues from actinobacteria. The source of the abbreviated Zurlike proteins is indicated by the respective GenBank identifiers. 


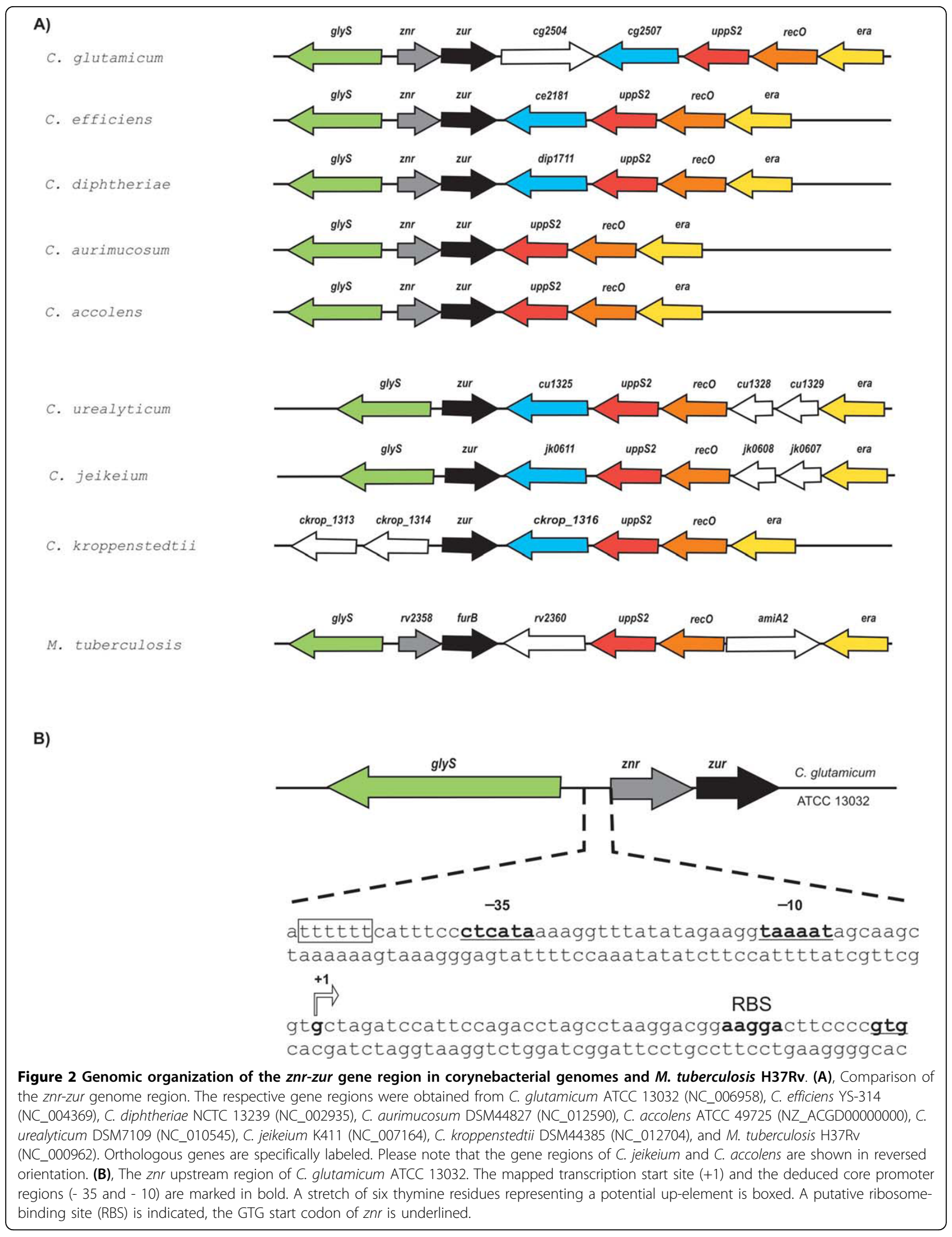


upstream region, indicating the presence of a promoter in front of $z n r$ and supporting the view that $z n r$ and $z u r$ are organized as operon. This observation was further strengthened by detecting with RT-PCR a 309-bp cDNA fragment that encompasses the intergenic region on the $z n r-z u r$ transcript (data not shown). The promoter in front of the $z n r$ gene was deduced from RACE-PCR experiments with total RNA purified from C. glutamicum ATCC 13032 cultures, showing that transcription starts at a guanine residue located 45 nucleotides upstream of the GTG start codon of znr (Fig. 2B). Based on the known consensus motif for corynebacterial promoters [29], potential -10 (TAAAAT) and -35 (CTCATA) promoter regions with an 18-bp spacing and a putative up-element [30] were detected (Fig. 2B).

\section{Computational identification of actinobacterial Zur} regulons

We applied comparative genomic techniques such as cross-genome comparison of shared regulatory sites [6] to reconstruct Zur regulons in the genomes of eight Corynebacterium species, as well as other representative members of the taxonomic class Actinobacteria (four Mycobacterium species, Propionibacterium acnes, Streptomyces coelicolor, Leifsonia xyli, Thermobifida fusca, and Bifidobacterium longum). Initially, we collected the upstream regions of candidate zinc uptake genes $(z n u A C B)$ in the analyzed actinobacterial genomes and applied the motif recognition program SignalX. The identified 21-bp palindromic motif (Fig. 4B) was similar to previously identified Zur-binding motifs in M. tuberculosis and S. coelicolor [31-33]. We constructed a positional-weight matrix for the identified Zur-binding motif and applied it to scan the genomes of actinobacteria for additional candidate Zur-binding sites. After filtering of false-positive sites by the consistency check approach and accounting for possible operon structures, we combined the final list of predicted members of the Zur regulons in the analyzed genomes of actinobacteria (see additional file 1) (Fig. 4A).

Overall, a conserved core of the reconstructed Zur regulons includes one or multiple paralogues of the zinc ABC-type transporter ZnuACB and a putative P-loop GTPase of the COG0523 family [34], orthologues of the B. subtilis YciC protein [35]. In Mycobacterium species, $P$. acnes, S. coelicolor, and L. xyli, the Zur regulon includes paralogues of various ribosomal proteins (RpmB, RpmG, RpmE, RpmF, RpmJ, RpsN, RpsR). These observations are in agreement with the previously described Zur-dependent regulation of ribosomal protein genes in M. tuberculosis and S. coelicolor [31-33]. The $z n r-z u r$ operon is preceded by a candidate Zurbinding site only in two Mycobacterium species (Fig. $4 \mathrm{~A})$. The $C$. diphtheriae Zur regulon includes the candidate $\mathrm{ABC}$-type metal transporter operon troA-sapD-
DIP0439-DIP0440-DIP0441-DIP0442 and the cmrA gene encoding a surface-associated protein [36]. Additional candidate Zur-binding sites were detected upstream of the $a d h A$ gene encoding zinc-dependent alcohol dehydrogenase in C. glutamicum [37] and adhA orthologues in C. accolens and C. diphtheriae (Fig. 4A).

\section{Global gene expression profiling of the zur-mutant $C$.} glutamicum JS2502

To identify C. glutamicum genes that are under transcriptional control by Zur, the zur gene was deleted in the chromosome of the wild-type strain C. glutamicum ATCC 13032 by an allelic exchange procedure, resulting in the mutant strain C. glutamicum JS2502. Growth of the zur-deficient mutant C. glutamicum JS2502 in minimal medium CGXII was indistinguishable from the parental wild-type strain (data not shown), indicating that deregulation of the Zur regulon is not detrimental to any basic physiological functions in C. glutamicum. The genome-wide expression profile of C. glutamicum JS2502 was compared with that of C. glutamicum ATCC 13032 by DNA microarray hybridizations. The resulting ratio/intensity $(m / a)$ plot of the normalized data, based on two hybridization experiments with label swapping, is presented in Fig. 5. By applying a ratio cutoff of \pm 1 , which is equivalent to relative expression changes of at least two-fold, 23 genes exhibited higher transcript levels in the zur mutant when compared to the wild-type strain, whereas three genes showed lower transcript levels in C. glutamicum JS2502.

Among the genes that are up-regulated in the zur mutant, we detected five C. glutamicum transcription units that are preceded by candidate Zur-binding sites: cg2911-cg2912-cg2913, cg0040-cg0041-cg0042/cg0043 and cg0794/cg0795 (Table 1). The first two operons encode components of the putative zinc/manganese ABC-type transporter $\mathrm{ZnuACB}$ and the putative secreted protein Cg0040, whereas the latter genes encode a P-loop GTPase of the COG0523 protein family (Cg0794 or $\mathrm{YciC}$ ) and a putative oxidoreductase of unknown physiological function (Cg0795), respectively. The adhA gene, encoding a zinc-dependent alcohol dehydrogenase [37] and predicted to be a candidate member of the Zur regulon with a conserved candidate Zur-binding site (AATTGAAAAACATTTCCATTA), was not detected as differentially expressed by DNA microarray hybridizations. In summary, genome-wide expression profiling and motif searches revealed six transcription units of $C$. glutamicum ATCC 13032 that were considered as potential targets for a direct transcriptional control by the zinc-sensing repressor Zur (Table 1).

The DNA microarray hybridization revealed 15 additional genes that were differentially expressed in the $z u r$ mutant C. glutamicum JS2502 (Table 2). As most of the corresponding $m$-values were close to the detection 


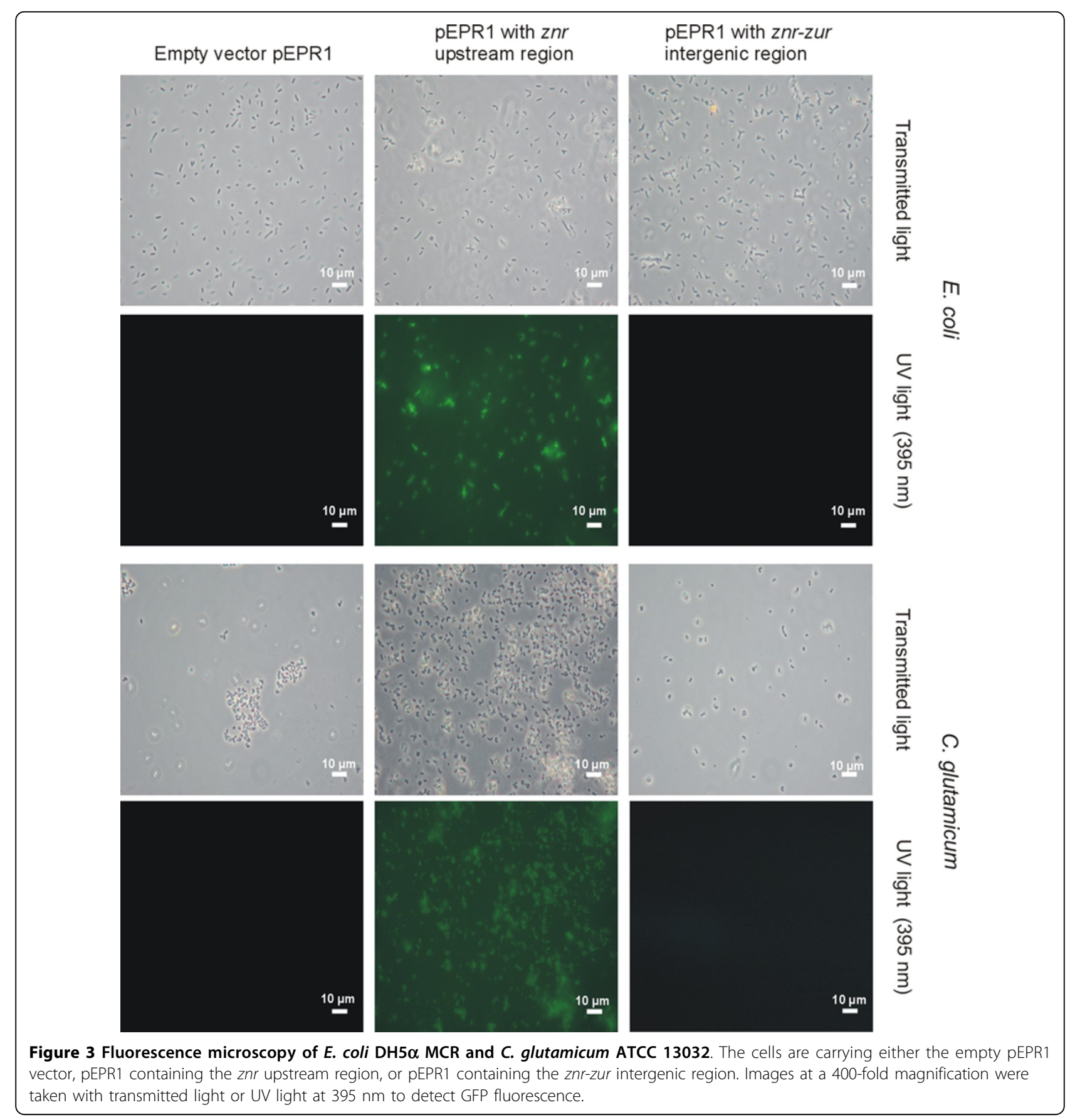

limit of the DNA microarray, expression of this gene set was furthermore examined by real-time RT-PCR. Using this more sensitive detection method, the expression of nine genes turned out to be significantly up-regulated in the zur mutant (Table 2). Among several coding regions of unknown function, this gene set includes cg1447 coding for a putative cobalt/zinc/cadmium efflux transporter and cg3096 (ald) encoding acetaldehyde dehydrogenase. In conjunction with the zinc-dependent alcohol dehydrogenase, the Ald protein is involved in the two-step utilization of ethanol as sole carbon and energy source by C. glutamicum [38]. As none of the genes is preceded by a candidate Zur-binding site, differential expression in C. glutamicum JS2502 is most likely a secondary effect of the zur gene deletion.

Verification of differential gene expression and promoter mapping

To support the conclusion that Zur is involved in transcriptional regulation of the potential target genes, control assays with a complemented C. glutamicum zur 


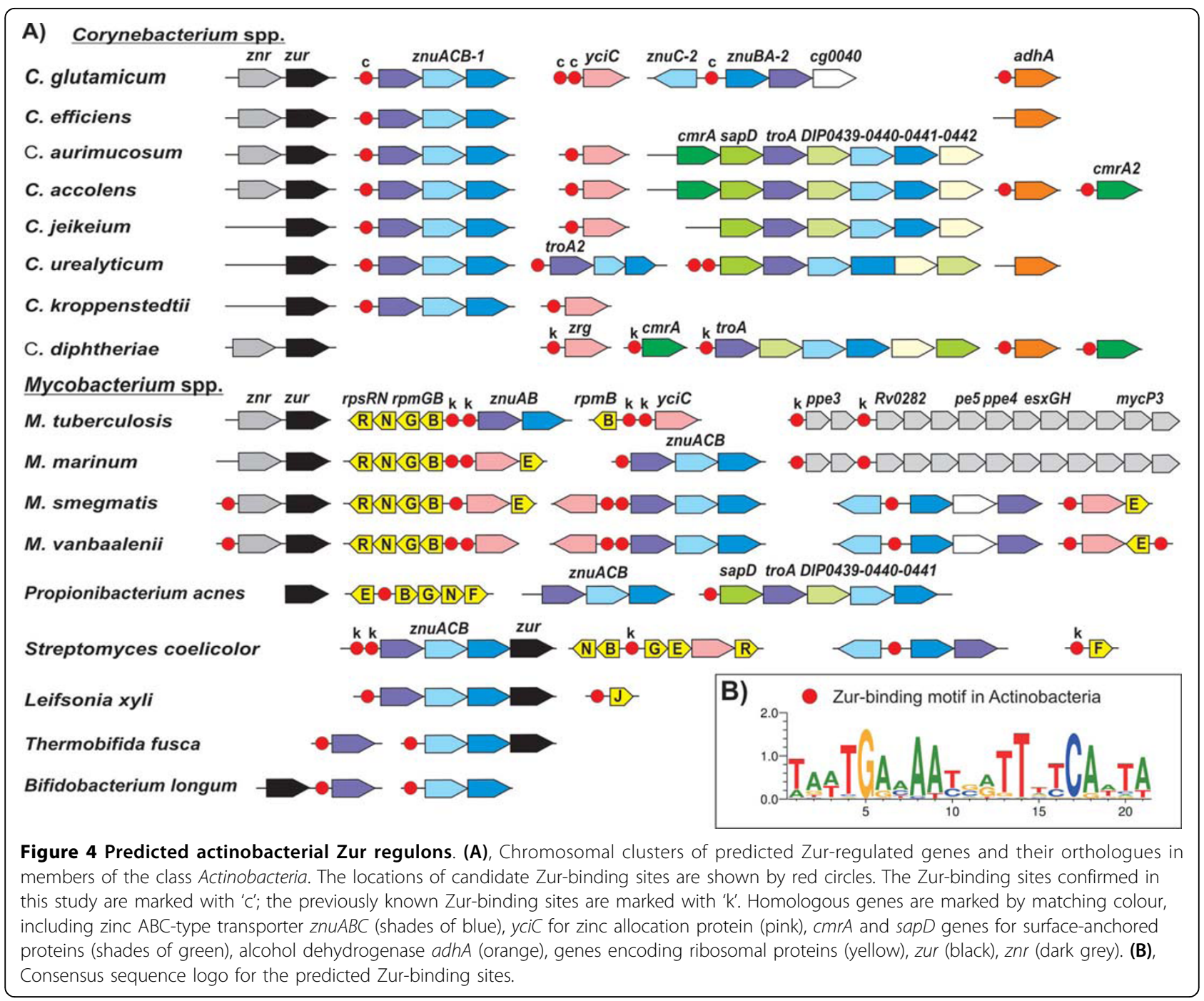

mutant were performed, thereby measuring the differential gene expression by RT-PCR. For this purpose, the $z u r$ gene was amplified by PCR and cloned into the $C$. glutamicum expression vector $\mathrm{pEC}-\mathrm{XK} 99 \mathrm{E}$, resulting in plasmid pEC-XK99E_zur (Table 3). First, the differential expression of potential Zur target genes in C. glutamicum JS2502 was verified by real-time RT-PCR assays. As expected, the mRNA levels of all genes were clearly enhanced in the zur mutant when compared with the wild-type strain (Table 1), with the exception of the adhA gene (data not shown). Additional RT-PCR assays with the complemented strain C. glutamicum JS2502 [pEC-XK99E_zur] showed that the expression of potential target genes was indistinguishable from that of the wild-type strain ATCC 13032 carrying the empty cloning vector pEC-XK99E (data not shown). These results clearly demonstrated that the observed deregulation of gene expression can be attributed to the defined deletion of the zur gene in C. glutamicum JS2502.
To elucidate whether the detected candidate Zurbinding motif is relevant for transcriptional regulation of the respective genes by Zur, the transcription start sites were determined by 5 ' RACE-PCR (Fig. 6). The mapped transcription sites were used to deduce thereof the respective promoter regions according to the corynebacterial consensus sequences for -10 and - 35 regions [29]. The transcription start sites in front of $c g 0042$, $\operatorname{cg} 0043$ and $\operatorname{cg} 2911$ were identical to the adenine residue of the respective ATG start codons, indicating the presence of so-called leaderless transcripts that were detected previously in C. glutamicum [29]. In all cases, the candidate Zur-binding motif overlaps the deduced core promoter regions (Fig. 6). Due to the short intergenic region (29 bp) between $c g 0042$ and $c g 0043$, a single candidate Zur-binding motif can be used to control the expression of the divergently oriented transcription units. The genetic organization of the cg0794-cg0795 intergenic region (118 bp) is more remarkable, as the 


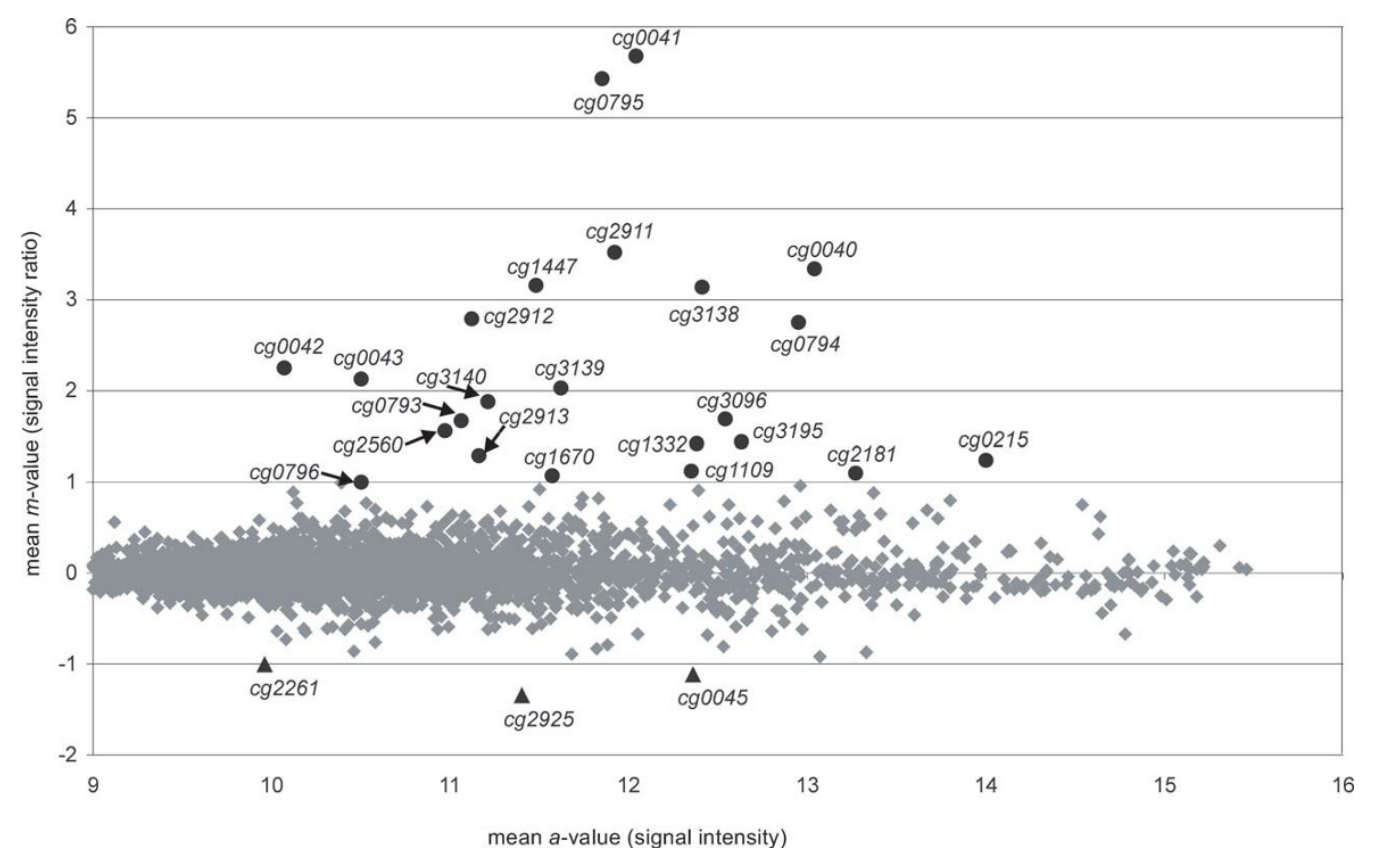

Figure 5 Ratio/intensity $(\mathrm{m} / \mathrm{a})$ plot deduced from DNA microarray hybridizations comparing the transcriptome of the zur mutant $C$. glutamicum JS2502 with that of the wild-type strain C. glutamicum ATCC 13032. Two biological replicates including label swapping were used for DNA microarray hybridizations. Genes showing significantly enhanced expression in C. glutamicum JS2502 are marked by black dots, decreased transcript levels are indicated by triangles, and genes without differential expression pattern are shown by grey diamonds. Genes were regarded as differentially expressed using the following cut-offs: $m$-value $\geq 1.0$, upregulation; $m$-value $\leq-1.0$, downregulation. The cut-offs correspond to relative changes in gene expression of at least two-fold.

motifs overlapping the - 35 region in front of cg0794 or cg0795 are both simultaneously located downstream of the -10 region belonging to the other gene (Fig. 6). These locations of the candidate Zur-binding motifs are consistent with the positions of operators used by repressor proteins to exert negative transcriptional control of gene expression [39]. As the Zur binding sites always overlap either the - 35 region or the entire - 10/-
35 region of its target promoters, Zur binding can block the entry of the RNA polymerase and thereby repress the transcription of the target genes. On the other hand, the candidate Zur-binding motif detected in the adhA gene region is located 167 nucleotides upstream of the mapped transcription start site [37]. These experimental data therefore indicated that five transcription units (cg0040-cg0041-cg0042/cg0043, cg2911-cg2912-cg2913,

Table 1 Differentially regulated Zur target genes preceded by candidate Zur-binding sites in C. glutamicum ATCC 13032.

\begin{tabular}{llllcc}
\hline CDS & Gene & Predicted function & 21-bp motif ${ }^{1}$ & \multicolumn{2}{c}{$\begin{array}{c}\text { Differential gene expression }^{2} \\
\text { Array }\end{array}$} \\
\hline cg0040 & - & secreted protein & - & 3.34 & 4.5 \\
cg0041 & znuA2 & ABC-type Zn/Mn transporter, substrate-binding protein & - & 5.68 & 27900 \\
cg0042 $^{3}$ & znuB2 & ABC-type Zn/Mn transporter, permease subunit & TAATGATAACGGTTATCATT & 2.25 & 331 \\
cg0043 & znuC2 & ABC-type Zn/Mn transporter, ATPase subunit & AAATGATAACCGTATCATTA & 2.13 & 50.2 \\
cg0794 & yciC & P-loop GTPase of the COG0523 family & TATTGAAAATGATTCCCAAAA & 2.75 & 10.5 \\
cg0795 & - & oxidoreductase & TAATGGAAATTGTTTCAATA & 5.43 & 45500 \\
cg2911 & znuA1 & ABC-type Zn/Mn transporter, substrate-binding protein & TGTTGACATCCTTTTCAATA & 3.52 & 43.8 \\
cg2912 & znuC1 & ABC-type Zn/Mn transporter, ATPase subunit & - & 2.79 & 75.8 \\
cg2913 & znuB1 & ABC-type Zn/Mn transporter, permease subunit & - & 1.29 & 29.0 \\
\hline
\end{tabular}

1 Genes listed without 21-bp motif (-) belong to predicted operons.

2 The gene expression in C. glutamicum JS2502 was compared with that of the wild-type strain ATCC 13032. Array, m-values obtained by DNA microarray

hybridizations (intensity ratio); RT-PCR, values obtained by RT-PCR (relative expression).

${ }^{3}$ First gene of the putative cg0042-cg0041-cg0040 operon.

${ }^{4}$ First gene of the putative cg2911-cg2912-cg2913 operon. 
Table 2 Differentially expressed genes in the zur mutant C. glutamicum JS2502 detected by DNA microarray hybridization and lacking candidate Zur-binding sites.

\begin{tabular}{|c|c|c|c|c|}
\hline \multirow[t]{2}{*}{ CDS } & \multirow[t]{2}{*}{ Gene } & \multirow[t]{2}{*}{ Predicted function } & \multicolumn{2}{|c|}{ Differential gene expression $^{1}$} \\
\hline & & & & \\
\hline $\mathrm{cg} 0045$ & - & ABC-type transporter, permease subunit & -1.12 & n.s. \\
\hline cg0215 & $\operatorname{csp} A$ & cold-shock protein A & 1.24 & n.s. \\
\hline cg0793 & - & putative secreted protein & 1.67 & 4.01 \\
\hline cg0796 & prpD1 & citrate dehydratase & 1 & 6.68 \\
\hline $\operatorname{cg} 1109$ & porB & anion-specific porin precursor & 1.12 & 7.67 \\
\hline $\operatorname{cg} 1332$ & - & putative secreted protein & 1.42 & 2.57 \\
\hline $\operatorname{cg} 1447$ & - & putative $\mathrm{Co}^{2+} / \mathrm{Zn}^{2+} / \mathrm{Cd}^{2+}$ efflux transporter & 3.16 & 25.8 \\
\hline $\operatorname{cg} 1670$ & - & hypothetical protein & 1.07 & 4.81 \\
\hline $\operatorname{cg} 2181$ & - & ABC-type transporter, substrate-binding protein & 1.1 & 4.91 \\
\hline $\operatorname{cg} 2261$ & $a m t B$ & secondary ammonium transporter & -1.01 & n.s. \\
\hline cg2560 & aceA & isocitrate lyase & 1.56 & n.s. \\
\hline cg2925 & ptss & phosphotransferase system component & -1.35 & n.s. \\
\hline cg3096 & ald & acetaldehyde dehydrogenase & 1.69 & 103 \\
\hline $\operatorname{cg} 3138$ & - & putative membrane protease subunit & 3.14 & n.s. \\
\hline cg3139 & - & hypothetical protein & 2.03 & n.s. \\
\hline $\operatorname{cg} 3140$ & $\operatorname{tag} A 1$ & DNA-3-methyladenine glycolase I & 1.88 & n.s. \\
\hline $\operatorname{cg} 3195$ & - & putative flavin-containing monooxygenase & 1.44 & 3.22 \\
\hline
\end{tabular}

${ }^{1}$ Gene expression in C. glutamicum JS2502 was compared with that of the wild-type strain ATCC 13032. Array, m-values obtained by DNA microarray hybridizations (intensity ratio); RT-PCR, values obtained by RT-PCR (relative expression).

${ }^{2}$ Abbreviation: n.s., no significant differences detected.

cg0794/cg0795) are under negative transcriptional regulation by Zur in C. glutamicum.

Verification of zinc-dependent expression of the putative cg0042 and cg2911 operons

As the putative operons $c g 0042$ and $c g 2911$ are apparently under negative control by the Zur protein in C. glutamicum, we investigated their zinc-dependent expression in vivo by using again the promoterless $g f p$ reporter system. For this purpose, the mapped promoter regions were amplified by PCR and cloned into the promoter-probe vector $\mathrm{pEPR} 1$. The resulting plasmids pEPR1_prom_cg0042 and pEPR1_prom_cg2911 (Table 3) were transferred into the C. glutamicum ATCC 13032 wild-type strain and into the $z u r$ mutant C. glutamicum JS2502 to detect differential $g f p$ expression by real-time RT-PCR, using high, low and chelated zinc conditions in the growth medium (Fig. 7). C. glutamicum ATCC 13032 carrying the empty cloning vector $\mathrm{pEPR} 1$ served as reference for calculating the differential gene expression. In the wild-type strain, the cloned promoters are apparently repressed under high-zinc condition and are derepressed under zinc-depletion, i.e. low-zinc condition and during growth in the presence of the chelator N,N,N',N'-tetrakis-(2-pyridylmethyl)-ethylenediamine (TPEN). A similar deregulation of gene expression was detected in the $z u r$ mutant C. glutamicum JS2502, irrespective of the presence or absence of zinc ions in the growth medium (Fig. 7 ). These in vivo data suggested that the lack of zincdependent regulation of gene expression is caused by the absence of the Zur protein in C. glutamicum JS2502. Furthermore, the data indicated that the Zur protein is sensing zinc ions and that it binds to operator sequences in the presence of zinc, thus acting as a repressor of the cg0042 and cg2911 operons.

Verification of predicted Zur binding sites by in vitro DNA band shift assays

To demonstrate experimentally the direct interaction of Zur with the candidate Zur-binding motifs detected in front of potential target genes, EMSAs were performed using fluorescein-labeled 40-mer oligonucleotides containing the 21-bp motif in the center of native genomic sequences (Fig. 8). For this purpose, the C. glutamicum Zur protein was tagged with streptavidin and purified by means of Strep-Tactin sepharose-packed columns (data not shown). Retardation of the respective doublestranded 40-mer DNA fragments was observed when the purified Zur protein and $50 \mu \mathrm{M} \mathrm{ZnCl}$ were added to the DNA band shift assays (Fig. 8A). In the absence of $\mathrm{ZnCl}_{2}$ no in vitro interaction of the purified Zur protein with the 40-mer DNA fragments was detected. A 40-mer sequence representing a regulatory gene region with a LexA binding site located in front of cg0841 [40] served as additional negative control. Likewise, Zur did not interact in vitro with the 21-bp motif located upstream of the $a d h A$ promoter region (Fig. 8A). Furthermore, mutated versions of the 21-bp motifs were generated by introducing transitions (Fig. 8B). In these cases, the purified Zur protein failed to shift the 

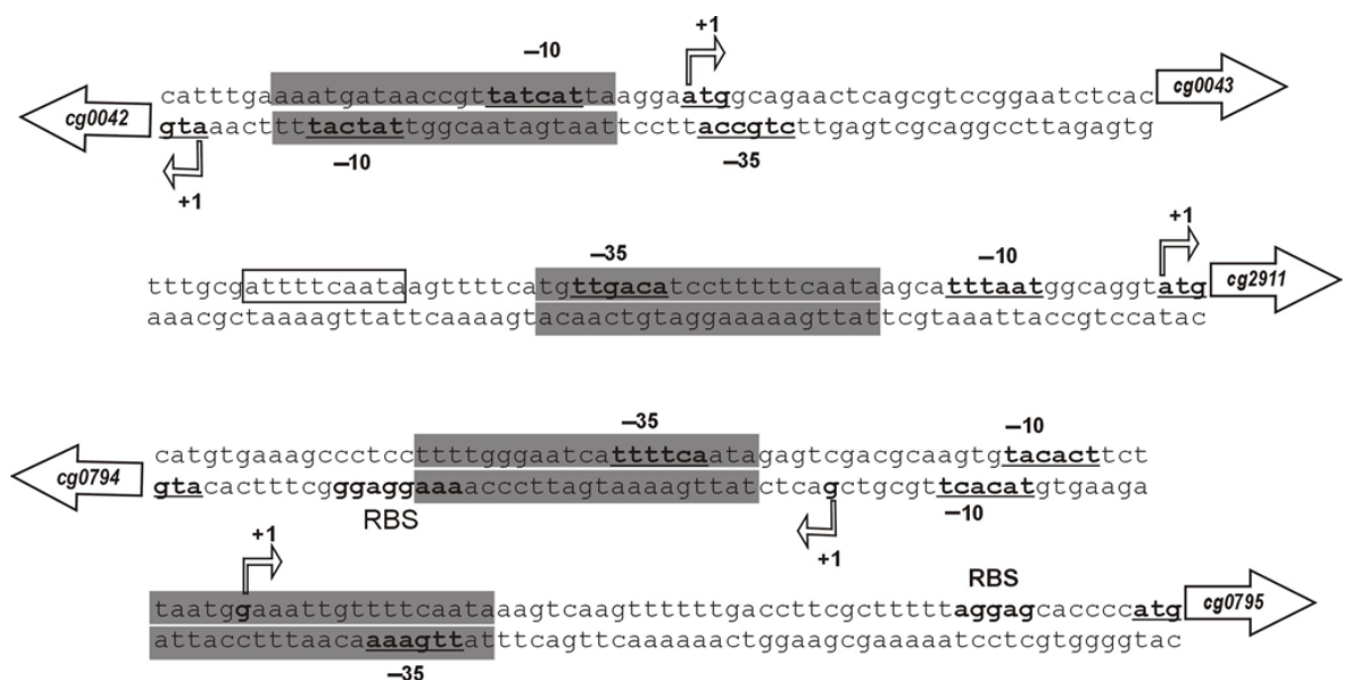

Figure 6 Promoter organization of the Zur regulon members in C. glutamicum ATCC 13032. A schematic presentation of relevant DNA regions from the C. glutamicum ATCC 13032 genome with detected promoters and candidate Zur-binding sites is presented. The 21-bp motifs are shown as grey boxes. A stretch of ten nucleotides (boxed), located upstream of the cg2911 promoter region, revealed similarity to the right half site of the 21-bp motif. The transcription start sites $(+1)$ were mapped by $5^{\prime}$ RACE-PCR and are marked in bold letters. Underlined nucleotides show the deduced -10 and -35 regions belonging to the corynebacterial promoters. Putative ribosome-binding sites (RBS) are indicated, start codons are underlined. The transcription start site and the - 10 region of cg0043 were deduced from bioinformatic predictions.

mutated DNA sequences. On the other hand, transitions introduced into the DNA segments flanking the 21-bp motifs did not affect the in vitro binding of Zur (Fig. $8 \mathrm{~B})$. To better define the role of metals in the ability of Zur to interact with its operators, EMSAs were performed in the presence of either $50 \mu \mathrm{M} \mathrm{ZnCl}_{2}, \mathrm{MgSO}_{4}$, $\mathrm{NiCl}_{2}, \mathrm{CuSO}_{4}, \mathrm{MnSO}_{4}$, or $\mathrm{FeSO}_{4}$ using exemplarily the 21-bp motif located upstream of $c g 2911$ (Fig. 8C). These assays showed that the purified Zur protein was able to interact with this DNA fragment in vitro in the presence of either zinc or manganese ions. Similar observations were reported from DNA binding assays with Zn-dependent regulators from $M$. tuberculosis [31] and B. subtilis [41]. The four 21-bp motifs recognized by the purified Zur protein in vitro were used to delineate their consensus sequence in C. glutamicum ATCC 13032, which is highly similar to the FurB (Zur) consensus binding site from $M$. tuberculosis that was defined experimentally by DNase I footprint analysis [31] (data not shown). In summary, these results demonstrated the specific interaction of the Zur protein with the 21-bp operator motif in the presence of zinc, thereby negatively controlling the expression of nine genes belonging to the Zur regulon in C. glutamicum ATCC 13032.

\section{Discussion}

The zur gene encoding a zinc uptake regulator is conserved in genomes of actinobacteria

In the present study, we have examined the regulatory role of the C. glutamicum Zur protein (Cg2502) in the direct transcriptional control of gene expression. Zur was classified by protein domain pattern analysis as member of the Zur subgroup of the Fur protein family [4]. Fur proteins form a ubiquitous group of metalresponsive transcription regulators in many diverse bacterial lineages [14,42-44]. Comparative genomics revealed the presence of more than one fur homologue in most members of the taxonomic class Actinobacteria whose genome sequences have been completely determined, indicating that a gene duplication event predated the appearance of the last common ancestor of the actinobacteria [45]. A corresponding evolutionary model suggested that the resulting paralogues maintained the main biochemical properties of the ancestor regulator, but became specialized for coordinating different metal ions $[45,46]$, including iron (Fur), manganese (Mur), nickel (Nur), and zinc (Zur) [14]. An apparent gene loss event occurred in the common ancestor of the corynebacteria, as Corynebacterium genomes do not contain the furA gene encoding a regulator for oxidative stress genes, but have the orthologous furB (zur) genes [45]. Accordingly, the zur gene product of $C$. glutamicum belongs to the small set of 24 transcription regulators that were detected in all hitherto sequenced corynebacterial genomes $[5,19]$. Moreover, synteny analyses revealed a conserved chromosomal region surrounding the zur gene in corynebacteria and other actinobacteria, including Mycobacterium, Nocardia and Rhodococcus species [45]. In these species, the zur gene is located downstream of another regulatory gene encoding a 


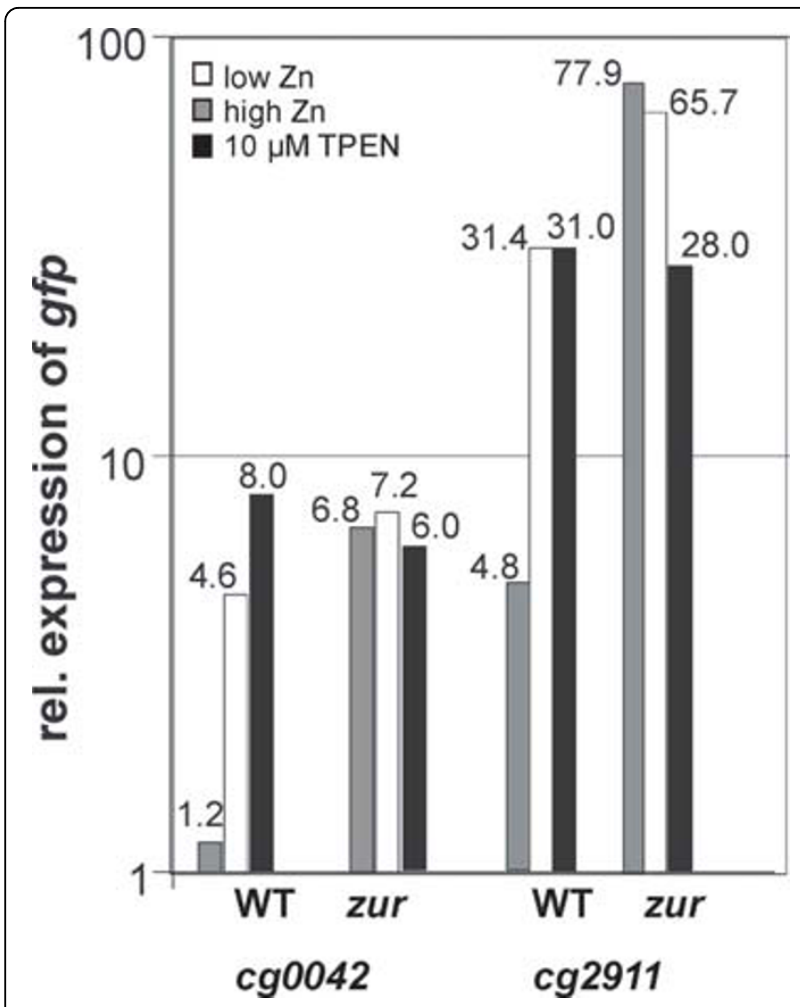

Figure 7 Zinc-dependent activity of the cg0042 and cg2911 operon promoters. The promoter activities of the Zur-regulated operons cg0042 and cg2911 was measured in the wild-type strain C. glutamicum ATCC 13032 (WT) and in the zur mutant C. glutamicum JS2502 (zur) under low, high and zinc-chelated (TPEN) conditions.

The relative expression of the gfp reporter gene was determined by real-time RT-PCR. The values are means of four measurements. The relative expression was calculated by using a C. glutamicum control carrying the empty expression vector pEPR1.

putative metal-sensing transcription regulator of the $\mathrm{SmtB} / \mathrm{ArsR}$ protein family $[10,45]$. Both regulators might be involved in controlling the balanced expression of genes involved in zinc uptake and metabolism in some actinobacteria [26,47]. In M. tuberculosis, the rv2358furB operon is (auto)regulated by Rv2358 [26] and functions as the regulatory interface between the control of zinc uptake and efflux [47]. At low zinc concentrations, Rv2358 negatively regulates expression of the $z i t A$ gene for a zinc efflux system [31] and the transcription of furB, thereby enabling the expression of FurB-regulated genes, including genes for zinc uptake systems [26]. At high zinc concentrations, Rv2358 does not bind to the operator site in front of the $r v 2358-f u r B$ operon and, as a consequence, zinc uptake is prevented by the regulatory action of FurB and an excess of zinc is pumped out of the cell. Since the genomic localization and the transcriptional organization of the znr-zur operon in C. glutamicum ATCC 13032 is similar to that of $M$. tuberculosis $\mathrm{H} 37 \mathrm{Rv}$, the regulatory role of $\mathrm{Cg} 2500$ (Znr) might be similar to that of Rv2358, i.e. both transcription regulators work together to optimally balance the zinc concentration in the $C$. glutamicum cell. To verify this conclusion, the target genes of $\mathrm{Znr}$ and its zincdependent interaction with the corresponding regulatory DNA sites have to be determined in C. glutamicum in future studies.

The set of genes differentially expressed in the zurmutant C. glutamicum JS2502 partially overlaps with the ethanol stimulon of $C$. glutamicum

The combination of genome-wide transcriptional profiling by DNA microarray hybridization and in vitro DNA band shift assays clearly demonstrated that the C. glutamicum Zur protein negatively controls the expression of five transcription units with genes that are involved in the zinc metabolism in this species. A comparison of the transcriptomes of the zur-deficient mutant C. glutamicum JS2502 and the wild-type strain C. glutamicum ATCC 13032 revealed 18 genes with increased expression in the zur mutant JS2502. This gene set, representing the cellular response to zur-deficiency in $C$. glutamicum JS2502, partially overlaps with a stimulon detected recently in C. glutamicum ATCC 13032 cells grown with ethanol as the sole carbon and energy source [38]. Growth of C. glutamicum ATCC 13032 on ethanol was characterized by enhanced expression levels of 36 genes when compared with acetate- and glucosegrown cultures. The set of differentially expressed genes detected in both genome-wide profiling studies include: (i) $c g 0040$ to $c g 0043$ and $c g 2911$ to $c g 2913$ encoding putative $A B C$-type uptake systems for zinc ions, (ii) cg3096 encoding acetaldehyde dehydrogenase and (iii) cg3195 encoding a putative flavoprotein. On the other hand, an enhanced expression of the Zur regulon members $c g 0794$ and $c g 0795$ was not deteced during growth of C. glutamicum on ethanol. The genes for the putative zinc uptake systems showed the largest increase of mRNA levels in ethanol-grown cells of C. glutamicum, which was explained by the higher demand of zinc due to its incorporation into the zinc-dependent alcohol dehydrogenase (AdhA) of C. glutamicum [38]. A candidate Zur-binding site was detected by cross-genome comparisons in the upstream region of adhA (cg3107), but the purified Zur protein did not bind to a corresponding 40-mer DNA sequence in vitro. In addition, the adhA gene was not detected as differentially expressed in the zur-deficient mutant C. glutamicum JS2502. Therefore, our results did not provide any evidence that the candidate Zur-binding site is involved in transcriptional regulation of $a d h A$ gene expression. The integration of the detected regulatory interactions into the database CoryneRegNet $[48,49]$ revealed that the Zur regulon forms a separate module in the transcriptional gene regulatory network model of C. glutamicum 
A)

cg0042

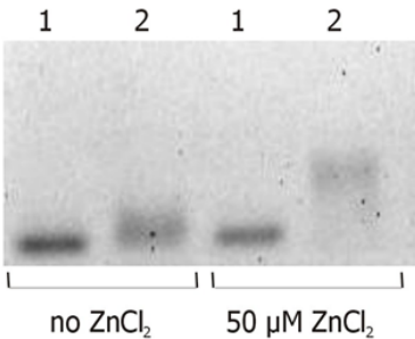

cg2911

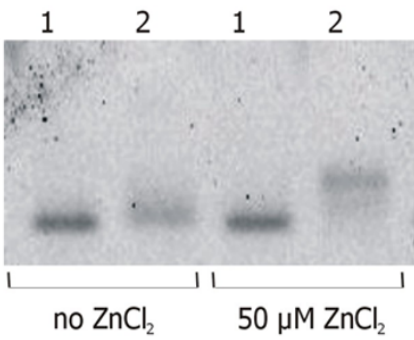

cg0794

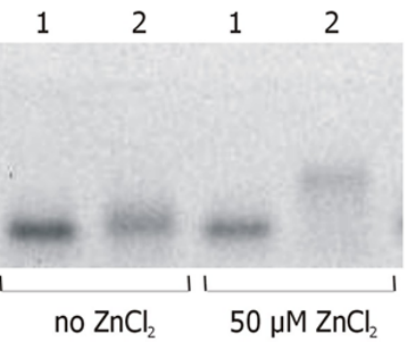

$\operatorname{cg} 3107(\mathrm{adh} A)$

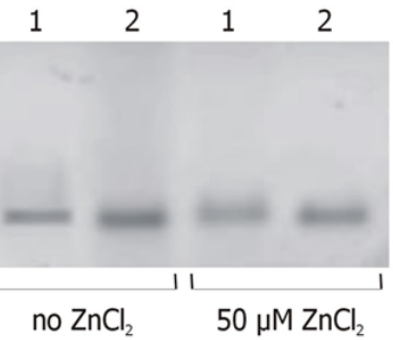

cg0795

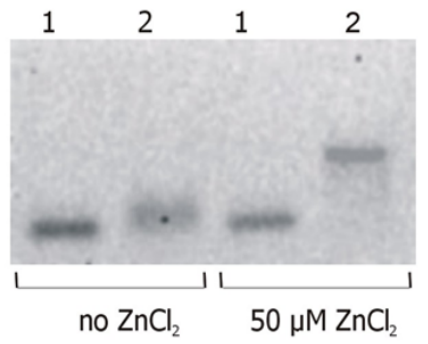

negative control (cg0841)

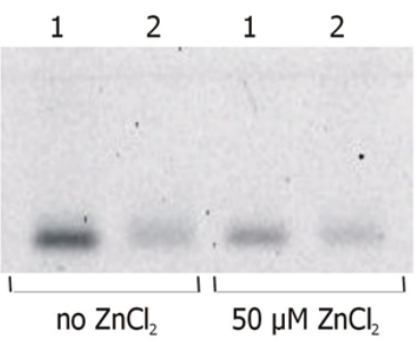

B)

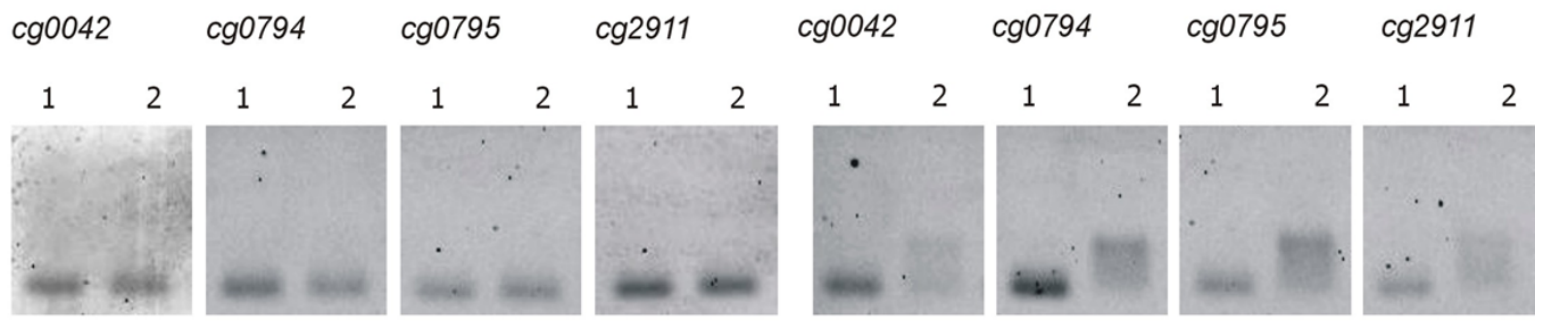

mutated binding sites

mutated flanking regions

C)

$\operatorname{cg} 2911$

no ions $\mathrm{Zn}^{2+} \quad \mathrm{Mg}^{2+} \quad \mathrm{Ni}^{2+} \quad \mathrm{Cu}^{2+} \quad \mathrm{Mn}^{2+} \quad \mathrm{Fe}^{2+}$

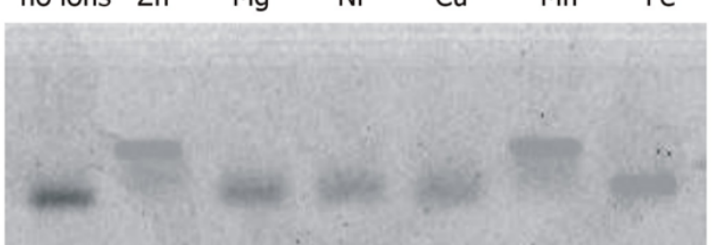

Figure 8 Agarose gels of DNA band shift assays with purified Zur protein. (A), The DNA band shift assays with fluorescein-labeled 40-mers covering the candidate Zur-binding sites in the cg0042-cg0043 intergenic region and in front of cg0794, cg0795, cg2911, and cg3107. DNA band shift assays were performed with 40 pmol of streptavidin-tagged Zur protein incubated with 0.05 pmol of fluorescein-labeled, double-stranded 40-mer DNA fragments. The assays were performed in the absence of zinc ions and in the presence of $50 \mu \mathrm{M} \mathrm{ZnCl}$. Lanes 1: control assays without Zur protein; lanes 2: DNA band shift assays with added Zur protein. The negative control assay was performed with a 40-mer deduced from the upstream region of cg0841. (B), DNA band shift assays with mutated versions of the 40-mers. Mutated versions were generated by introducing transitions into the candidate Zur-binding sites or into the genomic flanking regions. The EMSAs were carried out in the presence of $50 \mu \mathrm{M} \mathrm{ZnCl}$. (C), DNA band shift assays with binding buffers containing varying metal ions. The EMSAs were performed in the presence of 50 $\mu \mathrm{M} \mathrm{ZnCl}, \mathrm{MgSO}_{4}, \mathrm{NiCl}_{2}, \mathrm{CuSO}_{4}, \mathrm{MnSO}_{4}$, or $\mathrm{FeSO}_{4}$ with the 40-mer region representing cg2911. 
Table 3 Bacterial strains and plasmids used in this study.

\begin{tabular}{|c|c|c|}
\hline Strain or plasmid & Relevant characteristics & Source or reference \\
\hline C. glutamicum ATCC 13032 & wild-type strain & ATCC \\
\hline C. glutamicum JS2502 & ATCC 13032 with defined deletion in zur & This study \\
\hline E. coli DH5aMCR & E. coli strain used for standard cloning procedures & [73] \\
\hline E. coli TOP10 & E. coli strain used for cloning of RACE-PCR products & Invitrogen \\
\hline pCR2.1-TOPO & lacZ $\alpha, \mathrm{Ap}^{\mathrm{r}}$; E. coli cloning vector & Invitrogen \\
\hline pK18mobsacB & $s a c B, \mathrm{Km}^{\mathrm{r}}$; E. coli vector for allelic exchange & {$[74]$} \\
\hline pK18mobsacB_szur & $s a c B, \mathrm{Km}^{\mathrm{r}} ;$ pK18mobsacB carrying a modified zur gene with internal deletion & This study \\
\hline pASK-IBA5+ & $P_{\text {Tet, }}$ strep-tag, Ap ${ }^{r}$; E. coli expression vector & IBA Tagnologies \\
\hline pASK-IBA5+_cg2502 & pASK-IBA5+ carrying the C. glutamicum zur gene & This study \\
\hline pEPR1 & gfpuv ${ }_{P L}, \mathrm{Km}^{r}$; promoter-probe vector & {$[28]$} \\
\hline pEPR1_prom_cg2500 & gfpuv $v_{P L}, \mathrm{Km}^{r} ; \mathrm{pEPR} 1$ carrying the znr upstream region & This study \\
\hline pEPR1_prom_cg2502 & gfpuv $v_{P L}, \mathrm{Km}^{r} ; \mathrm{pEPR} 1$ carrying the zur upstream region & This study \\
\hline pEPR1_prom_cg0042 & gfpuv $_{P L}, \mathrm{Km}^{r} ;$ pEPR1 carrying the cg0042 upstream region & This study \\
\hline pEPR1_prom_cg2911 & gfpuv $_{\mathrm{PL},}, \mathrm{Km}^{r} ;$ pEPR1 carrying the cg2911 upstream region & This study \\
\hline pEC-XK99E & $\mathrm{P}_{\text {tra }}$ lacl, $\mathrm{Km}^{\mathrm{r}}$; C. glutamicum expression vector & {$[75]$} \\
\hline pEC-XK99E_zur & $P_{\text {tra }}$ lacl, Km; pEC-XK99E vector carrying the zur gene for complementation & This study \\
\hline
\end{tabular}

and is thus not linked to the currently known network supercluster [17]. Whether an additional carbon sourcedependent control of the Zur regulon by any kind of coregulation or hierarchical interaction is established in C. glutamicum remains to be elucidated.

\section{Physiological function of genes belonging to the Zur regulon of $C$. glutamicum}

Since the metal ions sensed by members of the Fur protein family are considered, on the one hand, fundamental for bacterial growth and, on the other hand, toxic at elevated levels, a strict balance between metal ion uptake and efflux is essential for homeostasis [14]. The target genes of the C. glutamicum Zur protein detected in this study include two putative ABC-type transport systems (Cg0041-Cg0043 and Cg2911-Cg2913), a putative secreted protein (Cg0040), a putative oxidoreductase (Cg0795), and a putative P-loop GTPase of the COG0523 family (Cg0794) that may specifically bind $\mathrm{Zn}^{2+}$ ions [50]. We also showed that Zur binds to the predicted operator sequences located in the mapped promoter regions of the respective genes, which are therefore under direct negative transcriptional control. The deduced genetic organization of the cg0794-cg0795 intergenic region and the common transcriptional control of both genes via two Zur operator sites suggests a functional link between the respective proteins. Since some experimentally characterized members of the COG0523 protein family of P-loop GTPases are socalled metallochaperones, such as HypB from Methanocaldococcus jannaschii [51] and UreG from Helicobacter pylori [52], the C. glutamicum P-loop GTPase Cg0794 may also function (eventually in conjuction with the oxidoreductase Cg0795) as a zinc-specific metallochaperone/insertase to enable the in vivo assembly of zinc- containing proteins under environmental conditions of zinc deficiency. Furthermore, Cg0794 is similar to YciC, an abundant protein from $B$. subtilis postulated to function as a metallochaperone [35]. Expression of $y c i C$ in $B$. subtilis occurs in a zinc-dependent manner that is exerted by the B. subtilis Zur orthologue [49]. YciC-like proteins are often members of the Zur regulons in proteobacteria and firmicutes and may be involved in the specific binding and allocation of $\mathrm{Zn}^{2+}$ ions [53]. The Cg2911 (ZnuA1) and Cg0041 (ZnuA2) proteins of $C$. glutamicum belong to the TroA superfamily of metalbinding proteins that are predicted to function as initial receptors in ABC-type transport systems of metal ions [21], supporting the view that both systems are involved in transport of divalent metal ions, such as $\mathrm{Zn}^{2+}$. The transcriptional regulation of genes encoding zinc uptake systems by Zur proteins seems to be common in actinobacteria, as the zur gene is located adjacent to $z n u$ operons in Arthrobacter, Leifsonia, Acidothermus, Nocardioides, Streptomyces, Thermobifida, and Rubrobacter species [45]. Likewise, genes encoding zinc ABCtype transport systems are under transcriptional control by Zur in Streptococcus suis [54], Xanthomonas campestris [55] and Yersinia pestis [56].

The transcriptional regulation of $z n u$ operons was characterized during genome-wide analyses of zinc-responsive regulators in M. tuberculosis H37Rv and Streptomyces coelicolor A3(2) [31-33]. The genes regulated by $\mathrm{Zur}_{M \text { tub }}$ encode three putative metal transporters, a group of ribosomal proteins and proteins belonging to the early secretory antigen target 6 (ESAT-6) cluster and the ESAT-6/ CFP-10 (culture filtrate protein 10) family [52]. Likewise, Zur $_{\text {Scoe controls the expression of } z n u A C B \text {, located }}$ upstream of zur and encoding a zinc uptake transporter, 
and of genes for paralogous forms of ribosomal proteins that are devoid of zinc-binding motifs and can therefore replace, during zinc deficiency, their zinc-binding counterparts that can serve as zinc storage forms [32,33]. Three DNA binding sites of $\mathrm{Zur}_{\text {Scoe }}$ were determined by DNase I footprinting analysis, revealing the 7-1-7 inverted repeat TGAAAATGATTTTCA as consensus sequence of potential operator sites [33]. This consensus sequence is similar to the central region of the 10-1-10 inverted repeat (candidate Zur-binding site) detected in the C. glutamicum genome in the present study. Likewise, DNA protection assays were used to identify Zur binding sites in the $M$. tuberculosis genome sequence [31]. The deduced 10-1-10 inverted repeat is also similar to the consensus sequence of Zur binding sites detected in the genome of C. glutamicum. Accordingly, the Zur binding sites in actinobacteria are apparently represented by a conserved 21-bp palindromic sequence with a 1-bp non-palindromic center, as shown by the Zur-binding motif sequence logo (Fig. 4B).

\section{Conclusions}

The combination of cross-genome comparison of shared regulatory sites and whole-genome expression profiling with DNA microarrays allowed us to deduce the Zur regulon of C. glutamicum ATCC 13032. It consists of five transcription units covering nine genes and encoding the components of two potential ZnuACB zinc transporters, a putative secreted protein, a putative oxidoreductase, and a putative P-loop GTPase of the COG0523 protein family. In vivo expression studies and in vitro DNA band shift assays demonstrated that Zur directly represses the expression of its target genes in a zinc-dependent manner. Accordingly, the Zur (Cg2502) protein is the key transcription regulator for genes involved in zinc homeostasis in C. glutamicum.

\section{Methods}

\section{Bacterial strains, plasmids and growth conditions}

Bacterial strains and plasmids used and constructed in this study are listed in Table 3. E. coli DH5 $\alpha$ MCR was grown at $37^{\circ} \mathrm{C}$ in Luria-Bertani medium [57] and used for standard cloning procedures as well as for heterologous expression of the C. glutamicum Zur protein. The induction of gene expression on the pASK-IBA5+ plasmid was carried out in E. coli DH5 $\alpha$ MCR using $200 \mathrm{ng} \mathrm{ml}^{-1}$ tetracycline. The wild-type strain C. glutamicum ATCC 13032 and the zur mutant C. glutamicum JS2502 were routinely grown at $30^{\circ} \mathrm{C}$ in CGXII minimal medium containing 30 $\mu \mathrm{g} \mathrm{l}^{-1}$ protocatechuic acid and $420 \mu \mathrm{g} \mathrm{l}^{-1}$ thiamine [58]. Antibiotics for plasmid selection were kanamycin $(50 \mu \mathrm{g}$ $\mathrm{ml}^{-1}$ for E. coli and $25 \mu \mathrm{g} \mathrm{ml}^{-1}$ for C. glutamicum) and ampicillin $\left(200 \mu \mathrm{g} \mathrm{ml}^{-1}\right.$ for E. coli). The growth of shakeflask cultures was monitored by measuring the optical density at $600 \mathrm{~nm}$ with an Eppendorf BioPhotometer.

\section{DNA preparation and PCR techniques}

The preparation of plasmid DNA from $E$. coli cells was performed by the alkaline lysis technique using the QIAprep Spin Miniprep Kit (Qiagen). The protocol was modified for C. glutamicum cells by using $20 \mathrm{mg} \mathrm{ml}^{-1}$ lysozyme in resuspension buffer P1 and by incubating the assay at $37^{\circ} \mathrm{C}$ for $3 \mathrm{~h}$. Chromosomal C. glutamicum DNA was isolated as described previously [59]. DNA restriction, analysis by agarose gel electrophoresis and DNA ligation were performed according to standard procedures [57]. The transformation of plasmid DNA was carried out by electroporation using electrocompetent $E$. coli and C. glutamicum cells $[60,61]$. The DNA amplification by PCR was performed with a PTC-100 thermocycler (MJ Research) and BIOTAQ DNA polymerase (Bioline) or Phusion Hot Start High-Fidelity DNA polymerase (Finnzymes). The PCR products were purified with the PCR Purification Spin Kit (Qiagen). All Oligonucleotides used in this study were purchased from Operon Biotechnologies (see additional file 2).

\section{Construction of a defined zur deletion in C. glutamicum}

The gene SOEing procedure [62] was applied to establish a defined deletion of 195 nucleotides in the zur coding region. The PCR primers used for gene SOEing were cg2502del1 to $c g 2502$ del4 (see additional file 2). The resulting pK18mobsacB derivative, pK18mobsacB_ $\Delta z u r$ (Table 3), was applied to perform an allelic exchange by homologous recombination in the chromosome of $C$. glutamicum ATCC 13032 [63], resulting in the mutant strain C. glutamicum JS2502. To complement the zur mutant phenotype, a DNA fragment covering the complete coding region of zur was amplified by PCR with the primer pair cg2502_compl1 and cg2502_compl2 (see additional file 2), digested with EcoRI and BamHI, and cloned in E. coli into the corresponding sites of shuttle expression vector pEC-XK99E (Table 3).

\section{Testing in vivo promoter activity}

The upstream region of the zur (cg2500) gene and the znr-zur intergenic region were amplified from chromosomal C. glutamicum DNA by PCR with the primer pairs cg2500_GFP1-cg2500_GFP2 and cg2502_GFP1cg2502_GFP2, respectively (see additional file 2). The PCR products were digested with appropriate enzymes and cloned into compatibel sites of the promoter-probe vector pEPR1 [28] that contains the promoterless $g f p$ reporter gene coding for the green fluorescent protein. The reporter gene of pEPR1 will be expressed only if the DNA fragment cloned in front of $g f p$ contains an active promoter [28]. The expression of the $g f p$ gene in E. coli DH5 $\alpha \mathrm{MCR}$ and C. glutamicum ATCC 13032 was detected by fluorescence microscopy with an Axiophot microscope (Zeiss) at a 400-fold magnification. All digital GFP pictures were taken with an exposure time of four seconds. 


\section{Measurement of in vivo promoter activity for cg0042 and cg2911}

To detect a zinc-dependent expression of the cg0042 and $c g 2911$ operons, approx. 200 bp segments covering the respective core promoter regions were amplified from chromosomal C. glutamicum DNA by PCR with the primer pairs cg0042_GFP1-cg0042_GFP2 and cg2911_GFP1-cg2911_GFP2, respectively (see additional file 2). The PCR products were cloned in E. coli $\mathrm{DH} 5 \alpha \mathrm{MCR}$ into the promoter-probe vector $\mathrm{pEPR} 1$, providing a promoterless $g f p$ reporter gene for subsequent measurements [28]. The plasmids were transformed into C. glutamicum ATCC 13032 and the zur mutant C. glutamicum JS2502 by electroporation. The resulting strains were grown in CGXII minimal medium containing $1 \mathrm{mg} \mathrm{l}^{-1} \mathrm{ZnSO}_{4}$ (high $\mathrm{Zn}$ condition) and in CGXII without additional $\mathrm{ZnSO}_{4}$ (low $\mathrm{Zn}$ condition). Additionally, the cells were exposed to $10 \mu \mathrm{M}$ of the chelator $\mathrm{N}$, N,N',N'-tetrakis-(2-pyridylmethyl)-ethylenediamine (TPEN) for $3 \mathrm{~h}$ in CGXII minimal medium (Zn-chelated condition). Expression of the $g f p$ reporter gene was measured by real-time RT-PCR using the primers LCPrimer1 $\_g f p$ and LCPrimer2_gfp (see additional file 2). RNA techniques and DNA microarray hybridizations

The isolation and purification of total RNA from C. $g l u$ tamicum cells was carried out as described previously [64]. The transcript levels of genes were measured by real-time reverse transcription PCR (RT-PCR) with the LightCycler instrument (Roche Applied Scince), using the SensiMix One-Step Kit (Quantace). The differences in gene expression between C. glutamicum JS2502 and the wild-type strain ATCC 13032 were determined by comparing the crossing points of two biological samples, each measured with two technical replicates. The measured crossing point (CP) is the cycle at which PCR amplification begins its exponential phase and is considered the point that is most reliably proportional to the initial RNA concentration (Roche Applied Science). The amounts of the mRNAs of the genes were normalized on total RNA, and the relative change in transcription rate was determined as $2-\Delta^{\mathrm{CP}}$, with $\Delta \mathrm{CP}$ equal to the difference of the measured crossing points for the test and the control condition. The crossing points were calculated by the LightCycler software (Roche Applied Science). The quality of the measurement was ensured by melting curve analysis.

Transcription start sites were determined by using the 5'/3' RACE Kit second generation according to the manufacturer's instructions (Roche Applied Science). Starting with $1 \mu \mathrm{g}$ of total C. glutamicum RNA, this approach enables the transcription of gene specific mRNA sequences into first-strand cDNA with the cDNA synthesis primer SP1 (see additional file 2). This initial cDNA synthesis was followed by a further amplification with nested PCR using the gene specific primer SP2 (see additional file 2). All PCR procedures were performed according to the recommendations of the manufacturer (Roche Applied Science) with a PTC100 thermocycler (MJ Research). The PCR products were cloned into the pCR2.1-TOPO vector using the TOPO TA Cloning Kit (Invitrogen), and the resulting plasmids were transferred into chemically competent $E$. coli TOP10 cells. The cloned RACE-PCR products were finally sequenced to determine the 5 ' end of the mRNA (IIT Biotech).

For global transcription profiling, hybridization of whole-genome DNA microarrays was performed with total RNA probes isolated from two independently grown $C$. glutamicum cultures. The respective cDNA samples were labeled with $\mathrm{Cy} 3 / \mathrm{Cy} 5$ in one experiment and with $\mathrm{Cy} 5 / \mathrm{Cy} 3$ in the other one (label swapping). Since each C. glutamicum DNA microarray contains four spots per gene, a maximum of eight spots per gene provided data for calculating differential gene expression. To minimize the number of false-positive signals, hybridization data were stringently filtered to obtain genes with at least six statistically significant values out of the eight technical replicates, applying an error probability of less than $5 \%$ for the $t$-test [64]. The data normalization was carried out with the LOWESS function, and $t$-test statistics were calculated with the EMMA2 software package [65]. The microarray hybridization data were deposited in the CoryneRegNet database with identifier "delta_zur" and can be downloaded for further analysis by using SOAP-based web services [66].

Overexpression and purification of the C. glutamicum Zur protein

To fuse the C. glutamicum Zur protein with an aminoterminal streptavidin (strep)-tag, the coding region of the zur gene was amplified by PCR with the primer pair cg2502_fwd_5Strep and cg2502_rev_5Strep (see additional file 2), which were created by using the IBA Primer D'Signer1.1 software (IBA BioTAGnology). The resulting PCR product was digested with BsaI and cloned into pASK-IBA5+ to give plasmid pASK-IBA5 +_cg2502 (Table 3) that was transferred to E. coli $\mathrm{DH} 5 \alpha$ MCR. Cell culturing, overexpression of the recombinant Zur protein and purification with Strep-Tactin sepharose-packed columns were carried out according to the manufacturer's instructions. The RiboLyser instrument was used for cell disruption, with a speed rate of 6.5 for two time intervals of $30 \mathrm{~s}$ and ice-cooling of $1 \mathrm{~min}$ inbetween. The concentration of the eluated protein was determined with the Bio-Rad protein assay kit (Bio-Rad Laboratories), and the eluate was analyzed by SDSPAGE. To verify the purification of the Zur protein, an in-gel digestion with modified trypsin (Promega) was 
carried out. A peptide mass fingerprint of the purified protein was determined by matrix-assisted laser desorption/ionization time-of-flight (MALDI-TOF) mass spectrometry, applying an Ultraflex mass spectrometer (Bruker Daltonics) and the MASCOT software.

\section{DNA band shift assays with streptavidin-tagged Zur} protein

Purified Zur protein was used in electrophoretic mobility shift assays (EMSAs) to determine its ability to interact with in silico predicted operators in dependence on zinc. EMSAs were performed using fluoresceinlabeled 40-mer oligonucleotides that were annealed with complementary oligonucleotides to double-stranded DNA fragments by heating for $5 \mathrm{~min}$ at $94^{\circ} \mathrm{C}$ and cooling on ice for $15 \mathrm{~min}$. The binding assays were performed in a final volume of $20 \mu \mathrm{l}$, containing $0.05 \mathrm{pmol}$ of the double-stranded 40-mer, 40 pmol of strep-tagged Zur protein, $0.06 \mu \mathrm{g}$ herring sperm DNA, and binding buffer $(20 \mathrm{mM}$ Tris- $\mathrm{HCl}, 50 \mathrm{mM} \mathrm{KCl}, 1 \mathrm{mM}$ DTT, 50 $\mu \mathrm{g} \mathrm{ml}^{-1}$ bovine serum albumin, $5 \%$ glycerol; $\mathrm{pH} 8.0$ ). EDTA was added to the binding reaction to a final concentration of $400 \mu \mathrm{M}$ [31]. Ions $\left(\mathrm{ZnCl}_{2}, \mathrm{MgSO}_{4}, \mathrm{NiCl}_{2}\right.$, $\mathrm{CuSO}_{4}, \mathrm{MnSO}_{4}$, or $\mathrm{FeSO}_{4}$ ) were added to EMSAs in a concentration of $50 \mu \mathrm{M}$. The assays were incubated at $30^{\circ} \mathrm{C}$ for $30 \mathrm{~min}$ and separated in $2 \%$ agarose gels prepared in gel buffer $(40 \mathrm{mM}$ Tris- $\mathrm{HCl}, 10 \mathrm{mM}$ sodium acetate, $1 \mathrm{mM}$ EDTA; pH 7.8). A voltage of $70 \mathrm{~V}$ was applied for $1 \mathrm{~h}$. The agarose gels were scanned with a Typhoon 8600 Variable Mode Imager (Amersham Biosciences Europe).

Bioinformatic methods and comparative genomic analysis of Zur regulons

The complete genomes of actinobacteria were downloaded from GenBank [67]. The Actinobacteria-specific training set for the identification of the Zur-binding motif was composed of the candidate zinc transporter genes $z n u A B C$. The DNA motif search profiles (a positional-weight matrix) were constructed using the SignalX program. Analyzed genomes were scanned with the constructed Zur-binding motif profile using the Genome Explorer software [68], and the identified genes with candidate Zur-binding sites were analyzed by the consistency check comparative procedure as previously described [6]. Positional nucleotide weights in the recognition profile and $Z$-scores of candidate sites were calculated as the sum of the respective positional nucleotide weights [69]. The threshold for the site search was defined as the lowest score observed in the training set $(Z$-score $=4.8)$. The sequence logo for the consensus Zur-binding motif in Actinobacteria was constructed using WebLogo 2.0 [70]. The phylogenetic trees were constructed by the maximum likelihood method implemented in the PROML program of the PHYLIP package [71] using multiple sequence alignments of protein sequences produced by the Clustal W2 program [72]. The deduced regulatory interactions were stored in the CoryneRegNet database [48].

Additional file 1: Candidate Zur-binding sites in the genomes of actinobacteria. The Excel file contains a list of detected Zur binding sites and candidate Zur-regulated genes.

Click here for file

[http://www.biomedcentral.com/content/supplementary/1471-2164-1112-S1.XLS ]

Additional file 2: Oligonucleotides used in this study. The PDF contains a list of all oligonucleotides used in the present work.

Click here for file

[http://www.biomedcentral.com/content/supplementary/1471-2164-1112-S2.PDF ]

\section{Acknowledgements}

The authors thank Eva Trost for providing data from the C. aurimucosum genome project prior to publication and Peter Heimann for help with fluorescence microscopy. The work of DAR was supported by a grant from the Russian Academy of Sciences (program "Molecular and Cellular Biology").

\section{Author details}

'Institut für Genomforschung und Systembiologie, Centrum für Biotechnologie, Universität Bielefeld, D-33615 Bielefeld, Germany. ${ }^{2}$ International NRW Graduate School in Bioinformatics and Genome Research, Centrum für Biotechnologie, Universität Bielefeld, D-33615 Bielefeld, Germany. ${ }^{3}$ Burnham Institute for Medical Research, La Jolla, CA 92037, USA. ${ }^{4}$ Institute for Information Transmission Problems (the Kharkevich Institute), RAS, 127994 Moscow, Russia.

\section{Authors' contributions}

JS performed the experimental work and drafted the manuscript. NJ participated in experimental design and data evaluation. DAR performed the genome-wide detection of Zur regulons in actinobacteria. AT participated in data evaluation and supervision. All authors read and approved the final version of the manuscript.

Received: 15 June 2009

Accepted: 7 January 2010 Published: 7 January 2010

\section{References}

1. Hermann T: Industrial production of amino acids by coryneform bacteria. J Biotechnol 2003, 104:155-172.

2. Leuchtenberger W, Huthmacher K, Drauz K: Biotechnological production of amino acids and derivatives: current status and prospects. Appl Microbiol Biotechnol 2005, 69:1-8.

3. Kalinowski J, Bathe B, Bartels D, Bischoff N, Bott M, Burkovski A, Dusch N, Eggeling L, Eikmanns BJ, Gaigalat L, et al: The complete Corynebacterium glutamicum ATCC 13032 genome sequence and its impact on the production of L-aspartate-derived amino acids and vitamins. J Biotechnol 2003, 104:5-25.

4. Brune I, Brinkrolf K, Kalinowski J, Pühler A, Tauch A: The individual and common repertoire of DNA-binding transcriptional regulators of Corynebacterium glutamicum, Corynebacterium efficiens, Corynebacterium diphtheriae and Corynebacterium jeikeium deduced from the complete genome sequences. BMC Genomics 2005, 6:86.

5. Brinkrolf K, Brune I, Tauch A: The transcriptional regulatory network of the amino acid producer Corynebacterium glutamicum. J Biotechnol 2007, 129:191-211

6. Rodionov DA: Comparative genomic reconstruction of transcriptional regulatory networks in bacteria. Chem Rev 2007, 107:3467-3497.

7. O'Halloran TV: Transition metals in control of gene expression. Science 1993, 261:715-725

8. Oram DM, Avdalovic A, Holmes RK: Analysis of genes that encode DtxRlike transcriptional regulators in pathogenic and saprophytic corynebacterial species. Infect Immun 2004, 72:1885-1895. 
9. Brown NL, Stoyanov JV, Kidd SP, Hobman JL: The MerR family of transcriptional regulators. FEMS Microbiol Rev 2003, 27:145-163.

10. Busenlehner LS, Pennella MA, Giedroc DP: The SmtB/ArsR family of metalloregulatory transcriptional repressors: Structural insights into prokaryotic metal resistance. FEMS Microbiol Rev 2003, 27:131-143.

11. Escolar L, Perez-Martin J, de Lorenzo V: Opening the iron box: transcriptional metalloregulation by the Fur protein. J Bacteriol 1999, 181:6223-6229.

12. Hantke K: Regulation of ferric iron transport in Escherichia coli K12: isolation of a constitutive mutant. Mol Gen Genet 1981, 182:288-292.

13. Hantke K: Iron and metal regulation in bacteria. Curr Opin Microbiol 2001, 4:172-177

14. Lee JW, Helmann JD: Functional specialization within the Fur family of metalloregulators. Biometals 2007, 20:485-499.

15. Blencowe DK, Morby AP: Zn(II) metabolism in prokaryotes. FEMS Microbiol Rev 2003, 27:291-311.

16. Patzer SI, Hantke K: The ZnuABC high-affinity zinc uptake system and its regulator Zur in Escherichia coli. Mol Microbiol 1998, 28:1199-1210.

17. Kohl TA, Baumbach J, Jungwirth B, Puhler A, Tauch A: The GlxR regulon of the amino acid producer Corynebacterium glutamicum: in silico and in vitro detection of DNA binding sites of a global transcription regulator. $J$ Biotechnol 2008, 135:340-350.

18. Brune I, Werner H, Hüser AT, Kalinowski J, Pühler A, Tauch A: The DtxR protein acting as dual transcriptional regulator directs a global regulatory network involved in iron metabolism of Corynebacterium glutamicum. BMC Genomics 2006, 7:21.

19. Tauch A, Schneider J, Szczepanowski R, Tilker A, Viehoever P, Gartemann KH, Arnold W, Blom J, Brinkrolf K, Brune I, et al: Ultrafast pyrosequencing of Corynebacterium kroppenstedtii DSM44385 revealed insights into the physiology of a lipophilic corynebacterium that lacks mycolic acids. J Biotechnol 2008, 136:22-30.

20. Gough J, Karplus K, Hughey R, Chothia C: Assignment of homology to genome sequences using a library of hidden Markov models that represent all proteins of known structure. J Mol Biol 2001, 313:903-919.

21. Marchler-Bauer A, Anderson JB, Derbyshire MK, DeWeese-Scott C, Gonzales NR, Gwadz M, Hao L, He S, Hurwitz DI, Jackson JD, et al: CDD: a conserved domain database for interactive domain family analysis. Nucleic Acids Res 2007, 35:D237-240.

22. Altschul SF, Madden TL, Schäffer AA, Zhang J, Zhang Z, Miller W, Lipman DJ: Gapped BLAST and PSI-BLAST: a new generation of protein database search programs. Nucleic Acids Res 1997, 25:3389-3402.

23. Lucarelli D, Russo S, Garman E, Milano A, Meyer-Klaucke W, Pohl E: Crystal structure and function of the zinc uptake regulator FurB from Mycobacterium tuberculosis. J Biol Chem 2007, 282:9914-9922.

24. Otsuka $Y$, Kawamura $Y$, Koyama T, lihara H, Ohkusu K, Ezaki T: Corynebacterium resistens sp. nov., a new multidrug-resistant coryneform bacterium isolated from human infections. J Clin Microbiol 2005, 43:37133717.

25. Pascual C, Lawson PA, Farrow JA, Gimenez MN, Collins MD: Phylogenetic analysis of the genus Corynebacterium based on 16S rRNA gene sequences. Int J Syst Bacteriol 1995, 45:724-728.

26. Canneva F, Branzoni M, Riccardi G, Provvedi R, Milano A: Rv2358 and FurB: two transcriptional regulators from Mycobacterium tuberculosis which respond to zinc. J Bacteriol 2005, 187:5837-5840.

27. Price MN, Huang KH, Alm EJ, Arkin AP: A novel method for accurate operon predictions in all sequenced prokaryotes. Nucleic Acids Res 2005 33:880-892.

28. Knoppova M, Phensaijai $M$, Vesely $M$, Zemanova $M$, Nesvera J, Patek M: Plasmid vectors for testing in vivo promoter activities in Corynebacterium glutamicum and Rhodococcus erythropolis. Curr Microbiol 2007, 55:234-239.

29. Pátek M, Nesvera J, Guyonvarch A, Reyes O, Leblon G: Promoters of Corynebacterium glutamicum. J Biotechnol 2003, 104:311-323.

30. Ross W, Ernst A, Gourse RL: Fine structure of $E$. coli RNA polymerasepromoter interactions: alpha subunit binding to the UP element minor groove. Genes Dev 2001, 15:491-506.

31. Maciag A, Dainese E, Rodriguez GM, Milano A, Provvedi R, Pasca MR, Smith I, Palu G, Riccardi G, Manganelli R: Global analysis of the Mycobacterium tuberculosis Zur (FurB) regulon. J Bacteriol 2007, 189:730740.
32. Owen GA, Pascoe B, Kallifidas D, Paget MS: Zinc-responsive regulation of alternative ribosomal protein genes in Streptomyces coelicolor involves zur and sigmaR. J Bacteriol 2007, 189:4078-4086.

33. Shin JH, Oh SY, Kim SJ, Roe JH: The zinc-responsive regulator Zur controls a zinc uptake system and some ribosomal proteins in Streptomyces coelicolor A3(2). J Bacteriol 2007, 189:4070-4077.

34. Tatusov RL, Natale DA, Garkavtsev IV, Tatusova TA, Shankavaram UT, Rao BS, Kiryutin B, Galperin MY, Fedorova ND, Koonin EV: The COG database: new developments in phylogenetic classification of proteins from complete genomes. Nucleic Acids Res 2001, 29:22-28.

35. Gabriel SE, Miyagi F, Gaballa A, Helmann JD: Regulation of the Bacillus subtilis yciC gene and insights into the DNA-binding specificity of the zinc-sensing metalloregulator Zur. J Bacterio/ 2008, 190:3482-3488.

36. Smith KF, Bibb LA, Schmitt MP, Oram DM: Regulation and activity of a zinc uptake regulator, Zur, in Corynebacterium diphtheriae. J Bacteriol 2009, 191:1595-1603.

37. Arndt A, Eikmanns BJ: The alcohol dehydrogenase gene adhA in Corynebacterium glutamicum is subject to carbon catabolite repression. $J$ Bacteriol 2007, 189:7408-7416.

38. Arndt $A$, Auchter $M$, Ishige $T$, Wendisch VF, Eikmanns BJ: Ethanol catabolism in Corynebacterium glutamicum. J Mol Microbiol Biotechnol 2008, 15:222-233.

39. Madan Babu M, Teichmann SA: Functional determinants of transcription factors in Escherichia coli: protein families and binding sites. Trends Genet 2003, 19:75-79.

40. Jochmann N, Kurze AK, Czaja LF, Brinkrolf K, Brune I, Huser AT, Hansmeier N, Puhler A, Borovok I, Tauch A: Genetic makeup of the Corynebacterium glutamicum LexA regulon deduced from comparative transcriptomics and in vitro DNA band shift assays. Microbiology 2009, 155:1459-1477.

41. Gaballa A, Helmann JD: Identification of a zinc-specific metalloregulatory protein, Zur, controlling zinc transport operons in Bacillus subtilis. $J$ Bacteriol 1998, 180:5815-5821.

42. Panina EM, Mironov AA, Gelfand MS: Comparative analysis of FUR regulons in gamma-proteobacteria. Nucleic Acids Res 2001, 29:5195-5206.

43. Panina EM, Mironov AA, Gelfand MS: Comparative genomics of bacterial zinc regulons: enhanced ion transport, pathogenesis, and rearrangement of ribosomal proteins. Proc Natl Acad Sci USA 2003, 100:9912-9917.

44. Rodionov DA, Dubchak I, Arkin A, Alm E, Gelfand MS: Reconstruction of regulatory and metabolic pathways in metal-reducing deltaproteobacteria. Genome Biol 2004, 5:R90.

45. Santos CL, Vieira J, Tavares F, Benson DR, Tisa LS, Berry AM, MoradasFerreira $P$, Normand $P$ : On the nature of fur evolution: a phylogenetic approach in Actinobacteria. BMC Evol Biol 2008, 8:185.

46. Rodionov DA, Gelfand MS, Todd JD, Curson AR, Johnston AW: Computational reconstruction of iron- and manganese-responsive transcriptional networks in alpha-proteobacteria. PLOS Comput Biol 2006, 2:e163.

47. Riccardi G, Milano A, Pasca MR, Nies DH: Genomic analysis of zinc homeostasis in Mycobacterium tuberculosis. FEMS Microbiol Lett 2008 287:1-7.

48. Baumbach J, Brinkrolf K, Czaja LF, Rahmann S, Tauch A: CoryneRegNet: an ontology-based data warehouse of corynebacterial transcription factors and regulatory networks. BMC Genomics 2006, 7:24

49. Baumbach J, Wittkop T, Kleindt CK, Tauch A: Integrated analysis and reconstruction of microbial transcriptional gene regulatory networks using CoryneRegNet. Nat Protoc 2009, 4:992-1005

50. Brown ED: Conserved P-loop GTPases of unknown function in bacteria: an emerging and vital ensemble in bacterial physiology. Biochem Cell Biol 2005, 83:738-746.

51. Gasper R, Scrima A, Wittinghofer A: Structural insights into HypB, a GTPbinding protein that regulates metal binding. J Biol Chem 2006, 281:27492-27502.

52. Zambelli B, Turano P, Musiani F, Neyroz P, Ciurli S: Zn2+-linked dimerization of UreG from Helicobacter pylori, a chaperone involved in nickel trafficking and urease activation. Proteins 2009, 74:222-239.

53. Haas CE, Rodionov DA, Kropat J, Malasarn D, Merchant SS, de CrecyLagard V: A subset of the diverse COG0523 family of putative metal chaperones is linked to zinc homeostasis in all kingdoms of life. BMC Genomics 2009, 10:470. 
54. Feng $Y$, Li M, Zhang H, Zheng B, Han H, Wang C, Yan J, Tang J, Gao GF: Functional definition and global regulation of Zur, a zinc uptake regulator in a Streptococcus suis serotype 2 strain causing streptococcal toxic shock syndrome. J Bacteriol 2008, 190:7567-7578.

55. Huang DL, Tang DJ, Liao Q, Li HC, Chen Q, He YQ, Feng JX, Jiang BL, Lu GT, Chen B, Tang JL: The Zur of Xanthomonas campestris functions as a repressor and an activator of putative zinc homeostasis genes via recognizing two distinct sequences within its target promoters. Nucleic Acids Res 2008, 36:4295-4309.

56. Li Y, Qiu Y, Gao H, Guo Z, Han Y, Song Y, Du Z, Wang X, Zhou D, Yang R: Characterization of Zur-dependent genes and direct Zur targets in Yersinia pestis. BMC Microbiol 2009, 9:128.

57. Sambrook J, Fritsch EF, Maniatis T: Molecular cloning: a laboratory manual., 21989.

58. Keilhauer C, Eggeling L, Sahm H: Isoleucine synthesis in Corynebacterium glutamicum: molecular analysis of the ilvB-ilvN-ilvC operon. J Bacteriol 1993, 175:5595-5603.

59. Tauch A, Kassing F, Kalinowski J, Pühler A: The Corynebacterium xerosis composite transposon Tn5432 consists of two identical insertion sequences, designated IS1249, flanking the erythromycin resistance gene ermCX. Plasmid 1995, 34:119-131.

60. Tauch A, Kirchner O, Wehmeier L, Kalinowski J, Pühler A: Corynebacterium glutamicum DNA is subjected to methylation-restriction in Escherichia coli. FEMS Microbiol Lett 1994, 123:343-347.

61. Tauch A, Kirchner O, Löffler B, Götker S, Pühler A, Kalinowski J: Efficient electrotransformation of Corynebacterium diphtheriae with a minireplicon derived from the Corynebacterium glutamicum plasmid pGA1. Curr Microbiol 2002, 45:362-367.

62. Horton RM, Hunt HD, Ho SN, Pullen JK, Pease LR: Engineering hybrid genes without the use of restriction enzymes: gene splicing by overlap extension. Gene 1989, 77:61-68.

63. Schäfer A, Tauch A, Jäger W, Kalinowski J, Thierbach G, Pühler A: Small mobilizable multi-purpose cloning vectors derived from the Escherichia coli plasmids pK18 and pK19: selection of defined deletions in the chromosome of Corynebacterium glutamicum. Gene 1994, 145:69-73.

64. Brune I, Jochmann N, Brinkrolf K, Hüser AT, Gerstmeir R, Eikmanns BJ, Kalinowski J, Pühler A, Tauch A: The IcIR-type transcriptional repressor $\mathrm{LtbR}$ regulates the expression of leucine and tryptophan biosynthesis genes in the amino acid producer Corynebacterium glutamicum. $J$ Bacteriol 2007, 189:2720-2733.

65. Dondrup M, Huser AT, Mertens D, Goesmann A: An evaluation framework for statistical tests on microarray data. J Biotechnol 2009, 140:18-26.

66. Baumbach J, Apeltsin L: Linking Cytoscape and the corynebacterial reference database CoryneRegNet. BMC Genomics 2008, 9:184.

67. Benson DA, Karsch-Mizrachi I, Lipman DJ, Ostell J, Sayers EW: GenBank. Nucleic Acids Res 2009, 37:D26-31.

68. Mironov AA, Vinokurova NP, Gel'fand MS: [Software for analyzing bacterial genomes]. Mol Biol (Mosk) 2000, 34:253-262.

69. Mironov AA, Koonin EV, Roytberg MA, Gelfand MS: Computer analysis of transcription regulatory patterns in completely sequenced bacterial genomes. Nucleic Acids Res 1999, 27:2981-2989.

70. Crooks GE, Hon G, Chandonia JM, Brenner SE: WebLogo: a sequence logo generator. Genome Res 2004, 14:1188-1190.

71. Felsenstein J: An alternating least squares approach to inferring phylogenies from pairwise distances. Syst Biol 1997, 46:101-111.

72. Larkin MA, Blackshields G, Brown NP, Chenna R, McGettigan PA, McWilliam H, Valentin F, Wallace IM, Wilm A, Lopez R, et al: Clustal W and Clustal X version 2.0. Bioinformatics 2007, 23:2947-2948.

73. Grant SG, Jessee J, Bloom FR, Hanahan D: Differential plasmid rescue from transgenic mouse DNAs into Escherichia coli methylation-restriction mutants. Proc Natl Acad Sci USA 1990, 87:4645-4649.

74. Schäfer A, Schwarzer A, Kalinowski J, Pühler A: Cloning and characterization of a DNA region encoding a stress-sensitive restriction system from Corynebacterium glutamicum ATCC 13032 and analysis of its role in intergeneric conjugation with Escherichia coli. J Bacteriol 1994 176:7309-7319.

75. Kirchner $\mathrm{O}$, Tauch A: Tools for genetic engineering in the amino acidproducing bacterium Corynebacterium glutamicum. J Biotechnol 2003, 104:287-299. doi:10.1186/1471-2164-11-12

Cite this article as: Schröder et al:: The Zur regulon of Corynebacterium glutamicum ATCC 13032. BMC Genomics 2010 11:12.

\section{Submit your next manuscript to BioMed Central and take full advantage of:}

- Convenient online submission

- Thorough peer review

- No space constraints or color figure charges

- Immediate publication on acceptance

- Inclusion in PubMed, CAS, Scopus and Google Scholar

- Research which is freely available for redistribution

Submit your manuscript at www.biomedcentral.com/submit
C Biomed Central 